\title{
The structure of molecular clumps around high-mass young stellar objects ${ }^{\star}$
}

\author{
F. Fontani ${ }^{1}$, R. Cesaroni ${ }^{2}$, P. Caselli ${ }^{2}$, and L. $\mathrm{Olmi}^{3}$ \\ 1 Dipartimento di Astronomia e Fisica dello spazio, Largo E. Fermi 2, 50125 Firenze, Italy \\ 2 INAF, Osservatorio Astrofisico di Arcetri, Largo E. Fermi 5, 50125 Firenze, Italy \\ 3 LMT/GTM Project, Dept. of Astronomy, 815J Lederle GRT Tower B, University of Massachusetts, \\ 710 N. Pleasant st., Amherst, MA 01003, USA
}

Received 14 December 2001 / Accepted 12 April 2002

\begin{abstract}
We have used the IRAM 30-m and FCRAO 14-m telescopes to observe the molecular clumps associated with 12 ultracompact (UC) HII regions in the $J=6-5,8-7$ and 13-12 rotational transitions of methyl-acetylene $\left(\mathrm{CH}_{3} \mathrm{C}_{2} \mathrm{H}\right)$. Under the assumption of LTE and optically thin emission, we have derived temperature estimates ranging from 30 to $56 \mathrm{~K}$. We estimate that the clumps have diameters of $0.2-1.6 \mathrm{pc}, \mathrm{H}_{2}$ densities of $10^{5}-10^{6} \mathrm{~cm}^{-3}$, and masses of $10^{2}-2 \times 10^{4} M_{\odot}$. We compare these values with those obtained by other authors from different molecular tracers and find that the $\mathrm{H}_{2}$ density and the temperature inside the clumps vary respectively like $n_{\mathrm{H}_{2}} \propto R^{-2.6}$ and $T \propto R^{-0.5}$, with $R$ distance from the centre. We also find that the virial masses of the clumps are $\sim 3$ times less than those derived from the $\mathrm{CH}_{3} \mathrm{C}_{2} \mathrm{H}$ column densities: we show that a plausible explanation is that magnetic fields play an important role to stabilise the clumps, which are on the verge of gravitational collapse. Finally, we show that the $\mathrm{CH}_{3} \mathrm{C}_{2} \mathrm{H}$ line width increases for decreasing distance from the clump centre: this effect is consistent with infall in the inner regions of the clumps. We conclude that the clumps around UC HiI regions are likely to be transient $\left(\sim 10^{5} \mathrm{yr}\right)$ entities, remnants of isothermal spheres currently undergoing gravitational collapse: the high mass accretion rates $\left(\sim 10^{-2} M_{\odot} \mathrm{yr}^{-1}\right)$ lead to massive star formation at the centre of such clumps.
\end{abstract}

Key words. stars: formation - radio lines: ISM - ISM: molecules

\section{Introduction}

While substantial progress has been done in recent years towards a better description of the formation of low-mass stars, much remains to be understood of their massive counterparts. In fact, several problems hinder the study of high-mass star formation: massive stars $\left(M \geq 10 M_{\odot}\right)$ are more distant, interact more strongly with their environment, and have shorter evolution timescales. After the protostellar phase, they reach the ZAMS and create an HII region in the circumstellar medium before the accretion phase is finished. When the star is very young (i.e. $\leq 10^{5} \mathrm{yr}$ ), this ionised region is small (i.e. $\leq 0.1 \mathrm{pc}$ for an $\mathrm{O}$ star) with respect to the typical diameter of an HII region associated with a more evolved OB star (i.e. 1$10 \mathrm{pc}$ ), and is named ultracompact (UC) His region. Given the strong interaction between early type stars and their

Send offprint requests to: F. Fontani,

e-mail: fontani@arcetri.astro.it

* Based on observations carried out with the IRAM Pico Veleta telescope. IRAM is supported by INSU/CNRS (France), MPG (Germany) and IGN (Spain). surroundings, it is important to have knowledge of the physical characteristics of their parental molecular clouds. With this in mind, Churchwell et al. (1990), Cesaroni et al. (1991), and Hofner et al. (2000) observed respectively $\mathrm{NH}_{3}, \mathrm{C}^{34} \mathrm{~S}$, and $\mathrm{C}^{17} \mathrm{O}$ towards a few UC Hit regions, thus assessing the existence of molecular clumps surrounding such UC HII regions. They estimated masses of $10^{3}$ $10^{4} M_{\odot}$, densities of $10^{4}-10^{5} \mathrm{~cm}^{-3}$, and diameters of $0.4-$ 1 pc. In another study, Olmi et al. (1993) detected various $\mathrm{CH}_{3} \mathrm{CN}$ rotational lines in a limited sample of UC HII regions, which demonstrated the existence of even hotter, denser cores inside the more extended clumps observed in the $\mathrm{C}^{34} \mathrm{~S}$ lines. Notwithstanding these results, a robust temperature estimate for the molecular clumps surrounding UC HII regions was still missing. In fact, on the one hand tracers such as $\mathrm{NH}_{3}$ and $\mathrm{C}^{17} \mathrm{O}$ provide temperature estimates based on $2-3$ transitions only and hence prone to relatively large uncertainties; on the other, $\mathrm{CH}_{3} \mathrm{CN}$ is an excellent "thermometer" but arises from a region much smaller and denser than the 1 pc clumps seen in $\mathrm{C}^{34} \mathrm{~S}$ and $\mathrm{C}^{17} \mathrm{O}$. Hence, the main goal of the present study was 
Table 1. List of the observed sources.

\begin{tabular}{rlccc}
\hline \hline \# & Source & $\begin{array}{c}\alpha(\mathrm{B} 1950) \\
(\mathrm{h} \mathrm{m} \mathrm{s})\end{array}$ & $\begin{array}{c}\delta(\mathrm{B} 1950) \\
\left({ }^{\circ}{ }^{\prime \prime}\right)\end{array}$ & $\begin{array}{c}v_{\mathrm{LSR}} \\
\left(\mathrm{km} \mathrm{s}^{-1}\right)\end{array}$ \\
\hline 1 & G5.89-0.39 & 175726.8 & -240356 & 10.0 \\
2 & G9.62+0.19 & 180316.2 & -203203 & 4.4 \\
3 & G10.47+0.03 & 180540.3 & -195221 & 67.8 \\
4 & G10.30-0.15 $\left(^{*}\right)$ & 180557.7 & -200626 & 13.5 \\
5 & G10.62-0.38 & 180730.7 & -195629 & -3.1 \\
6 & G19.61-0.23 & 182450.3 & -115833 & 41.6 \\
7 & G29.96-0.02 & 184327.1 & -024236 & 98.0 \\
8 & G31.41+0.31 & 184459.2 & -011607 & 97.0 \\
9 & G34.26+0.15 & 185046.1 & +011112 & 58.0 \\
10 & G45.47+0.05 & 191204.3 & +110411 & 62.0 \\
11 & W51D $\left(^{*}\right)$ & 192122.3 & +142515 & 60.0 \\
12 & IRAS 20126+4104 & 201241.0 & +410421 & -3.5 \\
\hline
\end{tabular}

$\left(^{*}\right)$ not observed with the FCRAO telescope.

to derive a good temperature estimate for the medium density molecular surroundings of UC HII regions.

The $\mathrm{CH}_{3} \mathrm{C}_{2} \mathrm{H}$ species is especially suitable to our purposes. This is a symmetric-top molecule (see Townes \& Schawlow 1975) with a small dipole moment $(\mu=0.75$ Debye, Dubrulle et al. 1978), which makes thermalisation easy even at densities as low as $10^{4} \mathrm{~cm}^{-3}$. We thus expect $\mathrm{CH}_{3} \mathrm{C}_{2} \mathrm{H}$ to be in "local thermodynamical equilibrium" (LTE) conditions in the clumps found by Cesaroni et al. (1991) around the UC HiI regions. Moreover, the large number of transitions observable in the same bandwidth makes the temperature estimate very accurate. For instance, Bergin et al. (1994) successfully used this method to measure the temperature of dense cores in giant molecular clouds, deriving temperatures of $\sim 40 \mathrm{~K}$.

In Sect. 2 we describe the observations and data reduction procedure, in Sect. 3 we summarise the observational results and derive the physical parameters of the clumps, in Sect. 4 we discuss the implications of the results obtained. The conclusions are drawn in Sect. 5 .

\section{Observations and data reduction}

\subsection{IRAM 30-m telescope}

The data from the IRAM 30-m telescope were obtained in the period from August 24 to 29, 1997. The source list is shown in Table 1 . The velocity of each source listed in Table 1 is taken from previous observations of various molecular transitions, and it is only an indicative value of the real velocity of the gas from which the $\mathrm{CH}_{3} \mathrm{C}_{2} \mathrm{H}$ lines arise. We simultaneously observed the (6-5), (87) and (13-12) rotational transitions of $\mathrm{CH}_{3} \mathrm{C}_{2} \mathrm{H}$ in the $3 \mathrm{~mm}, 2 \mathrm{~mm}$ and $1.3 \mathrm{~mm}$ bands respectively. The halfpower beam width (HPBW) of the IRAM 30-m telescope was $22^{\prime \prime}, 17^{\prime \prime}$, and $12^{\prime \prime}$ at $3 \mathrm{~mm}, 2 \mathrm{~mm}$ and $1.3 \mathrm{~mm}$, respectively.
Table 2. Spectrometers used for the IRAM 30-m observations.

\begin{tabular}{cccc}
\hline \hline Band & Spectrometer & $\begin{array}{c}\text { Channel Spacing } \\
(\mathrm{MHz})\end{array}$ & $\begin{array}{c}\text { Total Band } \\
(\mathrm{MHz})\end{array}$ \\
\hline \multirow{2}{*}{$3 \mathrm{~mm}$} & filterbank & 1.0 & 256 \\
& autocorrelator & 0.078 & 70 \\
$2 \mathrm{~mm}$ & filterbank & 1.0 & 256 \\
& autocorrelator & 0.078 & 70 \\
$1.3 \mathrm{~mm}$ & filterbank & 1.0 & 512 \\
& autocorrelator & 0.078 & 140 \\
\hline
\end{tabular}

The alignment between the different receivers was checked through continuum cross scans on Jupiter at the three frequencies of observation, and found to be accurate to within $4^{\prime \prime}$. Pointing was checked every $0.5-1$ hours by means of continuum cross scans on some UC HII regions of the Churchwell et al. (1990) sample. We believe it is accurate to within $4^{\prime \prime}$.

The spectrometers used simultaneously are an autocorrelator, which covered only the lowest $K$ components with high spectral resolution, and a filterbank with low spectral resolution, covering all $K$ lines for the (6-5) and (8-7) transitions and up to the $K=10$ component for the (13-12) transition. The main parameters of the spectrometers are listed in Table 2.

For all sources, the observations were made towards the nominal central positions given in Table 1. Only towards G31.41, we obtained two $3 \times 3$ point maps, one with $12^{\prime \prime}$ and the other with $24^{\prime \prime}$ spacing.

We observed using "wobbler switching", namely a nutating secondary reflector with a beam-throw of 240 " in azimuth and a phase duration of $2 \mathrm{~s}$. The data were calibrated with the chopper wheel technique (see Kutner \& Ulich 1981). We checked the calibration by measuring the intensities of two planets observed at the same frequencies as our sources. The main beam brightness temperature, $T_{\mathrm{MB}}$, and the flux density, $F_{\nu}$, are related by the expression $F_{\nu}(\mathrm{Jy})=4.7 T_{\mathrm{MB}}(\mathrm{K})$.

\subsection{FCRAO telescope}

Observations of $\mathrm{CH}_{3} \mathrm{C}_{2} \mathrm{H}$ (6-5) were carried out between March 16 and May 181997 with the Five College Radio Astronomy Observatory (FCRAO), located in New Salem (Massachusetts, USA). The receiver used was the QUARRY focal plane array (Erickson et al. 1992), which covered the $3 \mathrm{~mm}$ band $(90-116 \mathrm{GHz})$ and consisted of 15 separate beams disposed in a $3 \times 5$ configuration. The telescope HPBW at the frequency of the $\mathrm{CH}_{3} \mathrm{C}_{2} \mathrm{H}(6-5)$ line is $50^{\prime \prime}$. The calibration was carried out using the chopper wheel technique. The observations were made in position switching, moving the antenna in azimuth by $2^{\prime}$. The main beam brightness temperature, $T_{\mathrm{MB}}$, and the flux density, $F_{\nu}$, are related by the expression $F_{\nu}(\mathrm{Jy})=21.5 T_{\mathrm{MB}}(\mathrm{K})$.

The spectrometer used was an autocorrelator, with 512 channels and a spectral resolution of $80 \mathrm{kHz}$. At FCRAO 


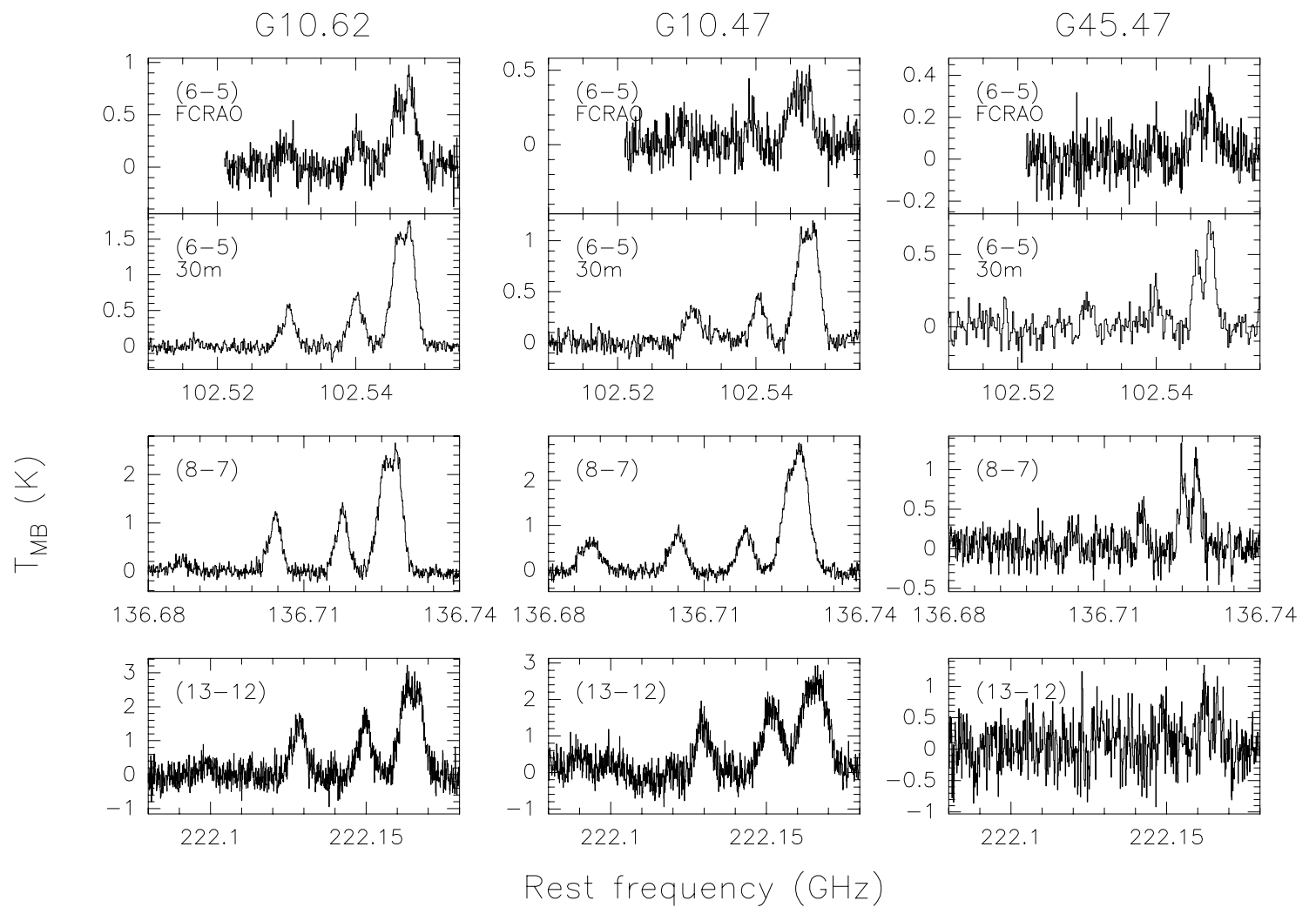

Fig. 1. Spectra of the $\mathrm{CH}_{3} \mathrm{C}_{2} \mathrm{H}(6-5)$, (8-7), and (13-12) rotational transitions towards sources G10.62, G10.47 and G45.47. On top, the FCRAO (6-5) spectra are shown.

we observed the same sample of UC HiI regions observed with the 30-m telescope, except G10.30 and W51D.

\subsection{Data reduction and fitting procedure}

$\mathrm{CH}_{3} \mathrm{C}_{2} \mathrm{H}$ is a symmetric-top molecule, with rotational levels described by two quantum numbers: $J$ is associated with the total angular momentum, whereas $K$ is associated with its projection on the symmetry axis. For each value of $J$, the selection rules $\Delta J= \pm 1$ and $\Delta K=0$ make possible only rotational radiative transitions $J+1 \rightarrow J$ with $K \leq J$; consequently, for each $J+1 \rightarrow J$ transition, only $J+1 K$-lines can be seen. In our observations we could detect only lines up to a maximum of $K=4$.

The data reduction made use of the CLASS program of the GAG software developed at IRAM and Observatoire de Grenoble. In order to derive the line parameters of $\mathrm{CH}_{3} \mathrm{C}_{2} \mathrm{H}$ we assumed that all the $K$ components of each $J+1 \rightarrow J$ transition arise from the same region, and are hence characterised by the same peak velocity and line width. Then, Gaussian fits to each $J+1 \rightarrow J$ spectrum were made with line separations between different $K$ components fixed to the laboratory values and line widths assumed to be identical.

\section{Results}

With the IRAM 30-m telescope we detected $\mathrm{CH}_{3} \mathrm{C}_{2} \mathrm{H}(6-$ $5),(8-7)$ and (13-12) towards all of the 12 sources of our sample, whereas with the FCRAO we detected $\mathrm{CH}_{3} \mathrm{C}_{2} \mathrm{H}$ (6-5) towards all of the sources but G19.61.

In Tables 3, 4, 5 and 6 we list the line parameters obtained with the data reduction procedure described in Sect. 2.3. In all cases, the difference between the line profile and the Gaussian fit was within the noise. For each source we list: the LSR velocity, $V_{\mathrm{LSR}}$; the line full width at half maximum (FWHM), $\Delta v_{1 / 2}$; the emission integrated under each line, $\int T_{\mathrm{MB}} \mathrm{d} v$; and the $1 \sigma \mathrm{rms}$ noise of the spectrum. For Gaussian profiles, the peak temperatures $T_{\mathrm{MB}}^{\max }$ are related to $\int T_{\mathrm{MB}} \mathrm{d} v$ by the relationship:

$$
\int T_{\mathrm{MB}} \mathrm{d} v=\frac{\Delta v_{1 / 2}}{2 \sqrt{\frac{\ln 2}{\pi}}} T_{\mathrm{MB}}^{\max } .
$$

The errors quoted do not take into account the calibration uncertainties, but represent only the formal errors of the fit procedure. The calibration errors will be discussed in Sect. 3.2.

\subsection{Size}

In order to assess that the $\mathrm{CH}_{3} \mathrm{C}_{2} \mathrm{H}$ lines originate from the 1 pc clumps known to exist around the UC HII regions, we must estimate the size of the $\mathrm{CH}_{3} \mathrm{C}_{2} \mathrm{H}$ emitting region. The maps obtained with the QUARRY multi-beam receiver on the FCRAO telescope are unsuitable to this purpose, because emission was detected only towards the central position. Consequently, only an upper limit to the 
Table 3. Parameters of the $\mathrm{CH}_{3} \mathrm{C}_{2} \mathrm{H}(6-5)$ lines observed with the IRAM 30-m telescope. The results given here are based on the autocorrelator (high resolution) spectra. The errors are given by the fit procedure, and do not consider the calibration error on $T_{\mathrm{MB}} . \Delta v_{1 / 2}$ represents the full width at half maximum (FWHM) of the line. $\sigma$ is the noise level in $\mathrm{K}$.

\begin{tabular}{|c|c|c|c|c|c|c|c|c|}
\hline \multirow[t]{2}{*}{ Source } & \multirow{2}{*}{$\begin{array}{c}v_{\mathrm{LSR}} \\
\left(\mathrm{km} \mathrm{s}^{-1}\right)\end{array}$} & \multirow{2}{*}{$\begin{array}{c}\Delta v_{1 / 2} \\
\left(\mathrm{~km} \mathrm{~s}^{-1}\right)\end{array}$} & \multicolumn{5}{|c|}{$\int T_{\mathrm{MB}} \mathrm{d} v\left(\mathrm{~K} \mathrm{~km} \mathrm{~s}^{-1}\right)$} & \multirow{2}{*}{$\begin{array}{c}\sigma \\
(\mathrm{K}) \\
\end{array}$} \\
\hline & & & $K=0$ & 1 & 2 & 3 & 4 & \\
\hline G5.89 & $8.54 \pm 0.08$ & $3.45 \pm 0.1$ & $15.15 \pm 0.1$ & $14.07 \pm 0.08$ & $7.24 \pm 0.1$ & $5.35 \pm 0.1$ & $0.85 \pm 0.1$ & 0.1 \\
\hline G9.62 & $4.41 \pm 0.02$ & $3.87 \pm 0.04$ & $7.45 \pm 0.07$ & $6.23 \pm 0.06$ & $2.82 \pm 0.06$ & $1.95 \pm 0.06$ & $0.23 \pm 0.06$ & 0.04 \\
\hline G10.47 & $66.66 \pm 0.02$ & $6.46 \pm 0.07$ & $6.77 \pm 0.1$ & $5.8 \pm 0.1$ & $2.94 \pm 0.1$ & $2.4 \pm 0.1$ & $0.24 \pm 0.1$ & 0.1 \\
\hline G10.30 & $13.06 \pm 0.05$ & $3.87 \pm 0.03$ & $2.18 \pm 0.05$ & $1.78 \pm 0.05$ & $0.81 \pm 0.05$ & $0.38 \pm 0.05$ & $0.08 \pm 0.05$ & 0.04 \\
\hline G10.62 & $-2.82 \pm 0.03$ & $6.13 \pm 0.06$ & $9.69 \pm 0.09$ & $8.41 \pm 0.08$ & $4.54 \pm 0.08$ & $3.28 \pm 0.08$ & $0.38 \pm 0.07$ & 0.06 \\
\hline G19.61 & $41.74 \pm 0.09$ & $6.32 \pm 0.06$ & $3.42 \pm 0.09$ & $2.93 \pm 0.09$ & $1.48 \pm 0.08$ & $1.10 \pm 0.07$ & $0.1 \pm 0.07$ & 0.05 \\
\hline G29.96 & $97.70 \pm 0.01$ & $2.75 \pm 0.03$ & $1.57 \pm 0.05$ & $1.40 \pm 0.04$ & $0.74 \pm 0.03$ & $0.59 \pm 0.04$ & $0.05 \pm 0.04$ & 0.05 \\
\hline G31.41 & $98.25 \pm 0.05$ & $4.31 \pm 0.05$ & $5.1 \pm 0.1$ & $3.8 \pm 0.1$ & $2.1 \pm 0.1$ & $1.6 \pm 0.1$ & $0.15 \pm 0.1$ & 0.1 \\
\hline G34.26 & $57.59 \pm 0.04$ & $5.38 \pm 0.06$ & $7.05 \pm 0.09$ & $6.64 \pm 0.08$ & $3.44 \pm 0.08$ & $2.18 \pm 0.08$ & $0.10 \pm 0.08$ & 0.09 \\
\hline G45.47 & $62.37 \pm 0.09$ & $3.87 \pm 0.05$ & $3.0 \pm 0.1$ & $2.0 \pm 0.1$ & $0.9 \pm 0.1$ & $0.75 \pm 0.1$ & $\leq 0.6$ & 0.1 \\
\hline W51D & $61.06 \pm 0.04$ & $4.30 \pm 0.06$ & $6.7 \pm 0.1$ & $5.7 \pm 0.1$ & $3.25 \pm 0.1$ & $2.8 \pm 0.1$ & $0.3 \pm 0.1$ & 0.1 \\
\hline IRAS 20126 & $-3.70 \pm 0.07$ & $2.18 \pm 0.02$ & $1.84 \pm 0.04$ & $1.52 \pm 0.04$ & $0.65 \pm 0.05$ & $0.39 \pm 0.04$ & $\leq 0.2$ & 0.04 \\
\hline
\end{tabular}

Table 4. Parameters of the $\mathrm{CH}_{3} \mathrm{C}_{2} \mathrm{H}(6-5)$ lines observed with the FCRAO telescope. G10.30 and W51D were not observed.

\begin{tabular}{|c|c|c|c|c|c|c|c|}
\hline \multirow[t]{2}{*}{ Source } & \multirow{2}{*}{$\begin{array}{c}v_{\mathrm{LSR}} \\
\left(\mathrm{km} \mathrm{s}^{-1}\right)\end{array}$} & \multirow{2}{*}{$\begin{array}{c}\Delta v_{1 / 2} \\
\left(\mathrm{~km} \mathrm{~s}^{-1}\right)\end{array}$} & \multicolumn{4}{|c|}{ 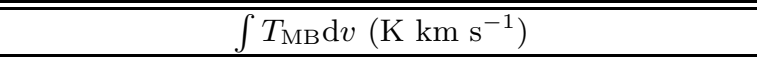 } & \multirow{2}{*}{$\begin{array}{c}\sigma \\
(\mathrm{K})\end{array}$} \\
\hline & & & $K=0$ & 1 & 2 & 3 & \\
\hline G5.89 & $8.79 \pm 0.05$ & $3.46 \pm 0.03$ & $6.7 \pm 0.2$ & $5.6 \pm 0.2$ & $3.0 \pm 0.2$ & $2.0 \pm 0.2$ & 0.2 \\
\hline G9.62 & $4.2 \pm 0.1$ & $4.2 \pm 0.1$ & $2.9 \pm 0.1$ & $2.6 \pm 0.1$ & $1.2 \pm 0.1$ & $0.6 \pm 0.1$ & 0.1 \\
\hline G10.47 & $66.9 \pm 0.2$ & $5.4 \pm 0.2$ & $2.2 \pm 0.2$ & $1.9 \pm 0.1$ & $0.9 \pm 0.1$ & $0.7 \pm 0.1$ & 0.09 \\
\hline G10.62 & $-2.9 \pm 0.1$ & $5.3 \pm 0.1$ & $4.3 \pm 0.2$ & $3.2 \pm 0.2$ & $1.5 \pm 0.2$ & $1.1 \pm 0.2$ & 0.1 \\
\hline G19.61(*) & - & - & $\leq 3.0$ & & & & 0.15 \\
\hline G29.96 & $97.8 \pm 0.2$ & $3.6 \pm 0.2$ & $1.05 \pm 0.09$ & $0.83 \pm 0.08$ & $0.29 \pm 0.08$ & $0.43 \pm 0.08$ & 0.1 \\
\hline G31.41 & $97.66 \pm 0.09$ & $4.2 \pm 0.1$ & $2.2 \pm 0.1$ & $1.9 \pm 0.1$ & $1.0 \pm 0.1$ & $0.8 \pm 0.1$ & 0.09 \\
\hline G34.26 & $57.52 \pm 0.04$ & $4.51 \pm 0.07$ & $3.79 \pm 0.07$ & $3.5 \pm 0.1$ & $1.5 \pm 0.1$ & $1.0 \pm 0.1$ & 0.1 \\
\hline G45.47 & $62.3 \pm 0.2$ & $4.7 \pm 0.3$ & $1.39 \pm 0.09$ & $1.12 \pm 0.09$ & $0.41 \pm 0.08$ & $\leq 0.3$ & 0.07 \\
\hline IRAS 20126 & $-3.68 \pm 0.06$ & $1.7 \pm 0.07$ & $0.58 \pm 0.03$ & $0.54 \pm 0.03$ & $0.22 \pm 0.02$ & $\leq 0.2$ & 0.05 \\
\hline
\end{tabular}

$\left({ }^{*}\right)$ Undetected source: the upper limits on $\int T_{\mathrm{MB}}$ have been obtained from $\sigma$ assuming a line FWHM equal to that measured with the IRAM 30-m telescope.

Table 5. Parameters of the $\mathrm{CH}_{3} \mathrm{C}_{2} \mathrm{H}(8-7)$ lines observed with the IRAM 30-m telescope.

\begin{tabular}{|c|c|c|c|c|c|c|c|c|}
\hline \multirow[t]{2}{*}{ Source } & \multirow{2}{*}{$\begin{array}{c}v_{\mathrm{LSR}} \\
\left(\mathrm{km} \mathrm{s}^{-1}\right)\end{array}$} & \multirow{2}{*}{$\begin{array}{c}\Delta v_{1 / 2} \\
\left(\mathrm{~km} \mathrm{~s}^{-1}\right)\end{array}$} & \multicolumn{5}{|c|}{$\int T_{\mathrm{MB}} \mathrm{d} v\left(\mathrm{~K} \mathrm{~km} \mathrm{~s}^{-1}\right)$} & \multirow{2}{*}{$\begin{array}{c}\sigma \\
(\mathrm{K})\end{array}$} \\
\hline & & & $K=0$ & 1 & 2 & 3 & 4 & \\
\hline G5.89 & $8.52 \pm 0.08$ & $3.50 \pm 0.05$ & $26.6 \pm 0.2$ & $25.3 \pm 0.2$ & $14.8 \pm 0.2$ & $12.2 \pm 0.2$ & $2.4 \pm 0.2$ & 0.2 \\
\hline G9.62 & $4.67 \pm 0.02$ & $3.67 \pm 0.06$ & $10.08 \pm 0.09$ & $8.34 \pm 0.09$ & $4.20 \pm 0.09$ & $2.99 \pm 0.08$ & $0.80 \pm 0.08$ & 0.07 \\
\hline G10.47 & $66.12 \pm 0.03$ & $7.5 \pm 0.1$ & $18.8 \pm 0.2$ & $11.7 \pm 0.2$ & $6.5 \pm 0.2$ & $6.2 \pm 0.2$ & $3.2 \pm 0.2$ & 0.2 \\
\hline G10.30 & $13.03 \pm 0.05$ & $3.85 \pm 0.03$ & $3.45 \pm 0.07$ & $2.80 \pm 0.07$ & $1.45 \pm 0.07$ & $0.90 \pm 0.07$ & $0.1 \pm 0.06$ & 0.08 \\
\hline G10.62 & $-2.92 \pm 0.03$ & $5.88 \pm 0.08$ & $14.2 \pm 0.1$ & $12.5 \pm 0.1$ & $7.7 \pm 0.1$ & $6.4 \pm 0.1$ & $1.4 \pm 0.1$ & 0.1 \\
\hline G19.61 & $41.19 \pm 0.08$ & $6.62 \pm 0.09$ & $6.2 \pm 0.1$ & $4.7 \pm 0.1$ & $2.8 \pm 0.1$ & $2.5 \pm 0.1$ & $1.0 \pm 0.1$ & 0.1 \\
\hline G29.96 & $97.69 \pm 0.01$ & $3.2 \pm 0.1$ & $3.45 \pm 0.07$ & $2.82 \pm 0.07$ & $1.52 \pm 0.07$ & $1.28 \pm 0.06$ & $0.18 \pm 0.06$ & 0.08 \\
\hline G31.41 & $97.74 \pm 0.03$ & $5.60 \pm 0.08$ & $10.50 \pm 0.2$ & $6.0 \pm 0.1$ & $3.8 \pm 0.1$ & $3.9 \pm 0.1$ & $1.1 \pm 0.1$ & 0.2 \\
\hline G34.26 & $57.67 \pm 0.04$ & $6.07 \pm 0.09$ & $16.0 \pm 0.1$ & $11.1 \pm 0.1$ & $6.1 \pm 0.1$ & $5.3 \pm 0.1$ & $1.1 \pm 0.1$ & 0.2 \\
\hline G45.47 & $62.49 \pm 0.09$ & $3.5 \pm 0.1$ & $4.0 \pm 0.2$ & $3.5 \pm 0.2$ & $1.8 \pm 0.2$ & $0.9 \pm 0.2$ & $\leq 0.7$ & 0.2 \\
\hline W51D & $60.84 \pm 0.04$ & $5.22 \pm 0.1$ & $15.0 \pm 0.2$ & $10.7 \pm 0.2$ & $7.3 \pm 0.2$ & $6.3 \pm 0.2$ & $1.7 \pm 0.2$ & 0.2 \\
\hline IRAS 20126 & $-3.77 \pm 0.07$ & $2.37 \pm 0.06$ & $3.025 \pm 0.07$ & $2.60 \pm 0.06$ & $1.10 \pm 0.06$ & $0.60 \pm 0.06$ & $0.06 \pm 0.05$ & 0.07 \\
\hline
\end{tabular}


Table 6. Parameters of the $\mathrm{CH}_{3} \mathrm{C}_{2} \mathrm{H}(13-12)$ lines observed with IRAM 30-m telescope.

\begin{tabular}{|c|c|c|c|c|c|c|c|c|}
\hline \multirow[t]{2}{*}{ Source } & \multirow{2}{*}{$\begin{array}{c}v_{\mathrm{LSR}} \\
\left(\mathrm{km} \mathrm{s}^{-1}\right)\end{array}$} & \multirow{2}{*}{$\begin{array}{c}\Delta v_{1 / 2} \\
\left(\mathrm{~km} \mathrm{~s}^{-1}\right)\end{array}$} & \multicolumn{5}{|c|}{$\int T_{\mathrm{MB}} \mathrm{d} v\left(\mathrm{~K} \mathrm{~km} \mathrm{~s}^{-1}\right)$} & \multirow{2}{*}{$\begin{array}{c}\sigma \\
(\mathrm{K})\end{array}$} \\
\hline & & & $K=0$ & 1 & 2 & 3 & 4 & \\
\hline G5.89 & $8.56 \pm 0.06$ & $3.92 \pm 0.06$ & $29.0 \pm 0.3$ & $28.6 \pm 0.3$ & $18.6 \pm 0.3$ & $18.0 \pm 0.3$ & $4.0 \pm 0.3$ & 0.4 \\
\hline G9.62 & $4.78 \pm 0.05$ & $4.16 \pm 0.06$ & $6.7 \pm 0.2$ & $6.0 \pm 0.2$ & $4.2 \pm 0.2$ & $3.6 \pm 0.2$ & $0.5 \pm 0.2$ & 0.2 \\
\hline G10.47 & $66.55 \pm 0.1$ & $8.0 \pm 0.1$ & $15.0 \pm 0.4$ & $16.0 \pm 0.2$ & $9.6 \pm 0.2$ & $9.5 \pm 0.1$ & $3.1 \pm 0.1$ & 0.4 \\
\hline G10.30 & $12.79 \pm 0.08$ & $3.47 \pm 0.09$ & $2.6 \pm 0.1$ & $2.4 \pm 0.1$ & $1.4 \pm 0.1$ & $1.0 \pm 0.1$ & $0.2 \pm 0.1$ & 0.2 \\
\hline G10.62 & $-2.74 \pm 0.05$ & $6.1 \pm 0.1$ & $14.6 \pm 0.3$ & $14.5 \pm 0.3$ & $9.6 \pm 0.3$ & $9.6 \pm 0.2$ & $2.2 \pm 0.2$ & 0.3 \\
\hline G19.61 & $40.8 \pm 0.1$ & $9.1 \pm 0.2$ & $5.7 \pm 0.3$ & $10.0 \pm 0.3$ & $6.5 \pm 0.2$ & $5.2 \pm 0.2$ & $0.4 \pm 0.2$ & 0.2 \\
\hline G29.96 & $98.01 \pm 0.05$ & $3.53 \pm 0.07$ & $3.1 \pm 0.1$ & $2.7 \pm 0.1$ & $1.7 \pm 0.1$ & $1.7 \pm 0.1$ & $0.2 \pm 0.1$ & 0.2 \\
\hline G31.41 & $97.96 \pm 0.1$ & $5.7 \pm 0.1$ & $10.62 \pm 0.1$ & $9.7 \pm 0.1$ & $9.5 \pm 0.1$ & $7.3 \pm 0.1$ & $2.1 \pm 0.1$ & 0.3 \\
\hline G34.26 & $58.28 \pm 0.05$ & $6.79 \pm 0.07$ & $12.1 \pm 0.3$ & $10.88 \pm 0.3$ & $7.9 \pm 0.2$ & $6.9 \pm 0.2$ & $1.8 \pm 0.2$ & 0.2 \\
\hline G45.47 & $62.9 \pm 0.2$ & $3.6 \pm 0.2$ & $2.5 \pm 0.3$ & $3.2 \pm 0.3$ & $2.2 \pm 0.3$ & $1.7 \pm 0.3$ & $\leq 1.1$ & 0.4 \\
\hline W51D & $60.59 \pm 0.06$ & $4.99 \pm 0.07$ & $16.0 \pm 0.2$ & $15.9 \pm 0.2$ & $10.0 \pm 0.1$ & $9.8 \pm 0.3$ & $1.6 \pm 0.3$ & 0.4 \\
\hline IRAS 20126 & $-3.44 \pm 0.1$ & $3.26 \pm 0.1$ & $1.9 \pm 0.1$ & $1.8 \pm 0.1$ & $0.8 \pm 0.1$ & $0.6 \pm 0.1$ & $0.1 \pm 0.1$ & 0.2 \\
\hline
\end{tabular}

source size could be obtained. However, another estimate of the size can be derived from the comparison between the $\mathrm{CH}_{3} \mathrm{C}_{2} \mathrm{H}(6-5)$ line intensities measured with the IRAM 30-m and FCRAO telescopes. Assuming Gaussian intensity distributions for the $\mathrm{CH}_{3} \mathrm{C}_{2} \mathrm{H}$ emitting clumps, one finds that the main beam brightness temperatures, $T_{\mathrm{MB}}$, measured with the two telescopes are related by the expression

$T_{\mathrm{MB}}^{\mathrm{fcrao}}\left(\Theta_{\text {fcrao }}^{2}+\Theta_{\mathrm{s}}^{2}\right)=T_{\mathrm{MB}}^{30 \mathrm{~m}}\left(\Theta_{30 \mathrm{~m}}^{2}+\Theta_{\mathrm{s}}^{2}\right)$

where $\Theta_{\mathrm{s}}$ is the FWHM of the source, and $\Theta_{30 \mathrm{~m}}$ and $\Theta_{\text {fcrao }}$ are the instrumental HPBWs.

From this equation one obtains the expression for $\Theta_{\mathrm{s}}$ :

$\Theta_{\mathrm{s}}^{2}=\frac{\Theta_{\mathrm{fcrao}}^{2}-\Theta_{30 \mathrm{~m}}^{2}\left(T_{\mathrm{MB}}^{30 \mathrm{~m}} / T_{\mathrm{MB}}^{\mathrm{fcrao}}\right)}{\left(T_{\mathrm{MB}}^{30 \mathrm{~m}} / T_{\mathrm{MB}}^{\mathrm{fcrao}}\right)-1}$.

The values of $\Theta_{\mathrm{s}}$ are listed in Table 7 and the corresponding linear diameters, $D$, are given in Table 9. The errors have been computed from Eq. (3) with the usual propagation of the statistical errors and hence depend upon the errors on $T_{\mathrm{MB}}$, which will be discussed in Sect. 3.2. The mean values are $\Theta_{\mathrm{s}} \simeq 30^{\prime \prime}$ and $D \simeq 0.9 \mathrm{pc}$. We stress that in all cases $\Theta_{\mathrm{s}}$ is consistent with the upper limits obtained from the FCRAO maps. Also, for G31.41 the value derived in this way can be compared with the direct estimate obtained from the emission map made with the IRAM 30-m telescope. Figures 2 and 3 show the maps of G31.41 obtained by integrating respectively the (6-5) and (8-7) emission under the $K=0$ and 1 lines. For a Gaussian source, one can compute the deconvolved clump diameter from the observed FWHM. We find $\Theta_{\mathrm{s}}=29^{\prime \prime}$ from the $\mathrm{CH}_{3} \mathrm{C}_{2} \mathrm{H}(6-5)$ map and $\Theta_{\mathrm{s}}=26^{\prime \prime}$ from $\mathrm{CH}_{3} \mathrm{C}_{2} \mathrm{H}(8$ $7)$. These estimates are consistent within the uncertainties with that obtained from Eq. (3), $\Theta_{\mathrm{s}}=32^{\prime \prime} \pm 8^{\prime \prime}$, thus confirming the reliability of the method. No size estimate can be derived from the $\mathrm{CH}_{3} \mathrm{C}_{2} \mathrm{H}(13-12)$ map because emission was detected only towards the central position: however, the upper limits obtained at offsets of $\pm 12^{\prime \prime}$ from the

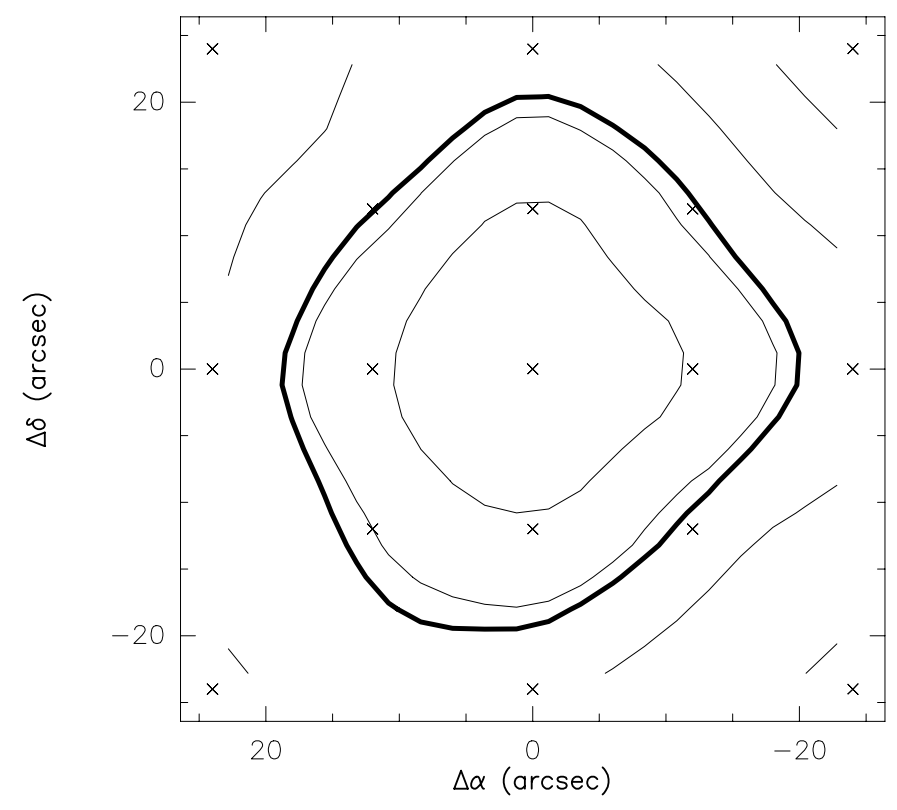

Fig. 2. Map obtained using the IRAM $30-\mathrm{m}$ telescope towards G31.41 by integrating the $\mathrm{CH}_{3} \mathrm{C}_{2} \mathrm{H}(6-5)$ emission under the $K=0$ and $K=1$ lines. Contour levels range from $1 \mathrm{~K} \mathrm{~km} \mathrm{~s}^{-1}$ (corresponding to $3 \sigma$ ) to $7 \mathrm{~K} \mathrm{~km} \mathrm{~s}^{-1}$ in steps of $2 \mathrm{~K} \mathrm{~km} \mathrm{~s}^{-1}$. The thick line corresponds to the half maximum power contour.

centre are consistent with the (13-12) line intensity measured towards the $(0,0)$ position and the source diameter estimated above.

We can also compare our results with those derived from $\mathrm{C}^{17} \mathrm{O}$ (Hofner et al. 2000) and $\mathrm{C}^{34} \mathrm{~S}$ (Cesaroni et al. 1991): in the former, the angular diameters are slightly greater $(\sim 20 \%)$ than those estimated by us, with the only exception of G10.47, which is two times larger in $\mathrm{C}^{17} \mathrm{O}$; in the latter tracer, instead, the angular sizes are $\sim 50 \%$ smaller with respect to $\mathrm{CH}_{3} \mathrm{C}_{2} \mathrm{H}$. We shall come back to this point in Sect. 4.2. 


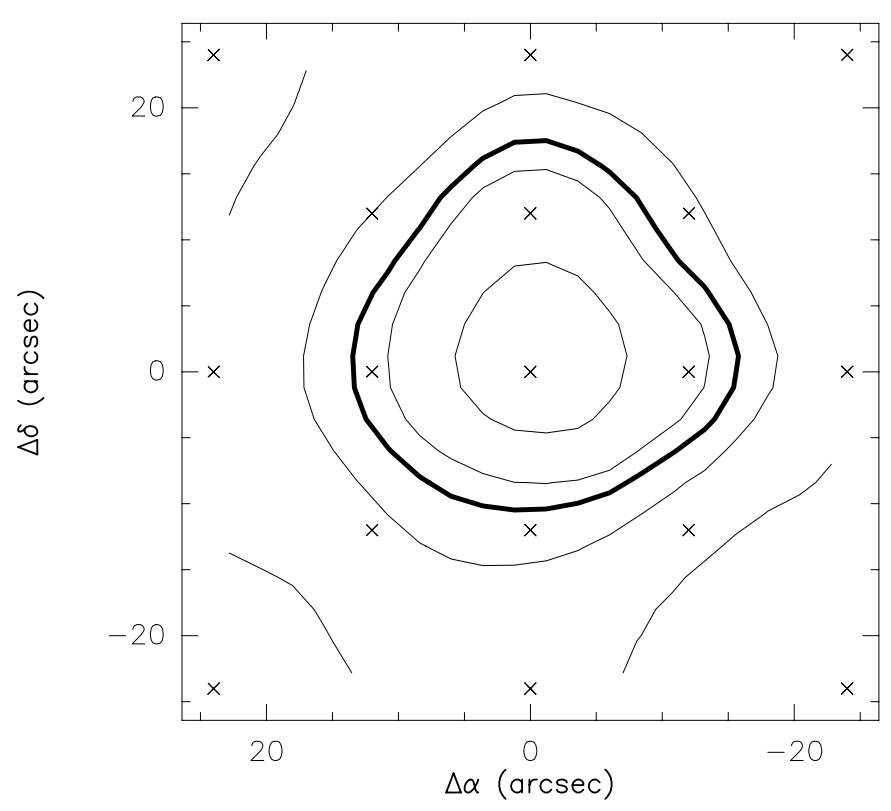

Fig. 3. Same as Fig. 2 for the $\mathrm{CH}_{3} \mathrm{C}_{2} \mathrm{H}\left(8^{-}-7\right) K=0$ and 1 lines. Contour levels range from $2 \mathrm{~K} \mathrm{~km} \mathrm{~s}^{-1}$ (corresponding to $3 \sigma$ ) to $18 \mathrm{~K} \mathrm{~km} \mathrm{~s}^{-1}$ in steps of $4 \mathrm{~K} \mathrm{~km} \mathrm{~s}^{-1}$.

\subsection{Temperature and column density}

From the angular diameters obtained from Eq. (3), we derived the rotation temperature and total column density of the $\mathrm{CH}_{3} \mathrm{C}_{2} \mathrm{H}$ molecules by means of the population diagram method (see e.g. Hollis 1982; Olmi et al. 1993), which assumes the gas to be in local thermodynamical equilibrium (LTE). Such an assumption is believed to work very well for $\mathrm{CH}_{3} \mathrm{C}_{2} \mathrm{H}$ (see Bergin et al. 1994) due to its low dipole moment. Under the additional assumption of optically thin emission, one can compute the beam averaged column density $N_{\mathrm{i}}$ of the upper level $i$ from the line intensity:

$\frac{N_{\mathrm{i}}}{g_{i}}=\frac{3 k \int T_{\mathrm{MB}} \mathrm{d} v}{8 \pi^{3} \eta_{\nu} \mu^{2} \nu S}$

where $k$ is the Boltzmann constant, $\eta_{\nu}$ the beam filling factor, $\nu$ the line rest frequency, $S$ the line strength, $g_{i}$ the statistical weight, and $\mu=0.75$ Debye (Dubrulle et al. 1978) the dipole moment of the $\mathrm{CH}_{3} \mathrm{C}_{2} \mathrm{H}$ molecule.

In Table 7 we give the rotational temperatures $\left(T_{\text {rot }}\right)$ and total $\mathrm{CH}_{3} \mathrm{C}_{2} \mathrm{H}$ source column densities $\left(N_{\text {tot }}\right)$ : these have been obtained from a least square fit to the data, as shown in the "Boltzmann plots" in Fig. 4. We note that each column density $N_{\mathrm{i}}$ in Fig. 4 includes the correction for angular resolution effects, assuming gaussian profiles for both the instrumental beam and the source. Therefore, $N_{\text {tot }}$ corresponds to a mean value over the clumps. The temperatures are distributed between 30 and $50 \mathrm{~K}$ and the column densities are of order $10^{14}-10^{15} \mathrm{~cm}^{-2}$. The goodness of the fits in Fig. 4 confirms that the gas is very close to LTE, which in turn implies that the rotational temperature derived in this way is an excellent estimate of the kinetic temperature, $T_{\mathrm{k}}$, of the molecular gas.

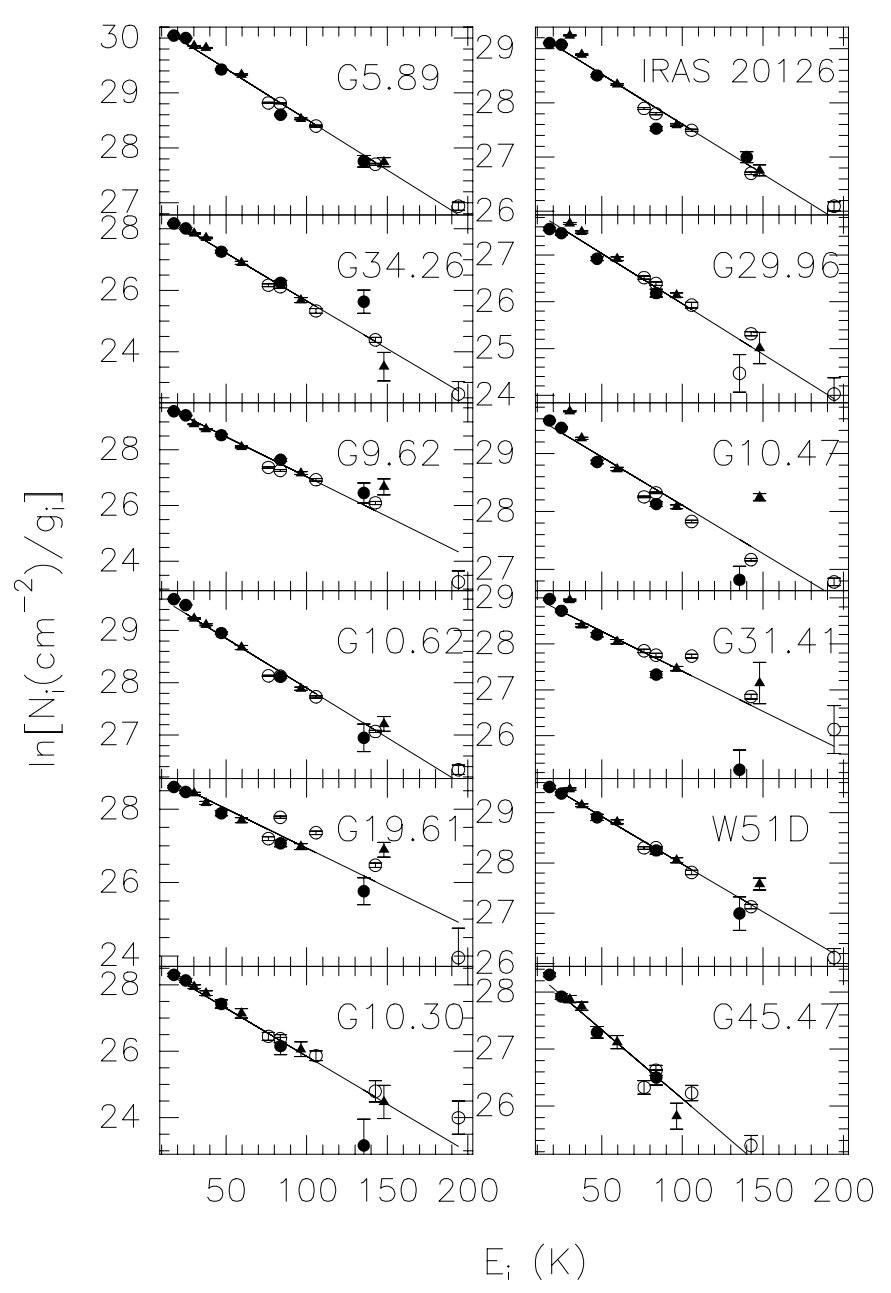

Fig. 4. Boltzmann plots for all of the observed sources. Filled circles represent the $\mathrm{CH}_{3} \mathrm{C}_{2} \mathrm{H}(6-5)$ data, triangles the (8-7), and empty circles the (13-12). The column densities have been obtained from the IRAM 30-m spectra. The straight lines represent least square fits to the data. The error bars correspond to the formal errors of the fitting procedure and do not consider the calibration error on $T_{\mathrm{MB}}$.

The errors quoted in Table 7 take into account calibration uncertainties on $T_{\mathrm{MB}}$ of $10 \%$ for the $3 \mathrm{~mm}$ lines and $20 \%$ for the others. For $T_{\text {rot }}$ and $N_{\text {tot }}$, the uncertainties have been estimated allowing for a variation of the values of $N_{\mathrm{i}}$ by $10 \%$ simultaneously for all of the $\mathrm{CH}_{3} \mathrm{C}_{2} \mathrm{H}(6-$ $5) \mathrm{K}$ lines and by $20 \%$ for the (8-7) and (13-12) lines: this takes into account the fact that each sample of $J+1 \rightarrow J$ transitions is affected by the same calibration uncertainty. Then the errors have been taken equal to the largest difference between the values of $T_{\text {rot }}$ and $N_{\text {tot }}$ thus obtained and the nominal ones.

As previously explained, the population diagram method uses the assumptions of LTE and optically thin emission: let us now discuss these in some better detail.

The LTE hypothesis is acceptable when the $\mathrm{H}_{2}$ density in the clump is greater than the critical density (see e.g. Spitzer 1978). The maximum critical density for the transitions observed by us is $\sim 10^{5} \mathrm{~cm}^{-3}$ : we shall see in the 
Table 7. Angular diameters, rotation temperatures, and source averaged column densities of the $\mathrm{CH}_{3} \mathrm{C}_{2} \mathrm{H}$ clumps. The errors are calculated assuming a calibration error of $\sim 10 \%$ at $3 \mathrm{~mm}$ and $\sim 20 \%$ at $2 \mathrm{~mm}$ and $1.3 \mathrm{~mm}$ (see text).

\begin{tabular}{lccc}
\hline \hline Source & $\begin{array}{c}\Theta_{\mathrm{s}} \\
(\operatorname{arcsec})\end{array}$ & $\begin{array}{c}T_{\text {rot }} \\
(\mathrm{K})\end{array}$ & $\begin{array}{c}N_{\text {tot }} \\
\left(10^{14} \mathrm{~cm}^{-2}\right)\end{array}$ \\
\hline G5.89 & $31 \pm 8$ & $48 \pm 10$ & $20 \pm 1$ \\
G9.62 & $28 \pm 7$ & $34 \pm 6$ & $13 \pm 1$ \\
G10.47 & $22 \pm 5$ & $46 \pm 8$ & $27 \pm 1$ \\
G10.30 $\left(^{*}\right)$ & 25 & $34 \pm 7$ & $5 \pm 1$ \\
G10.62 & $27 \pm 8$ & $46 \pm 10$ & $21 \pm 1$ \\
G19.61 $\left(^{*}\right)$ & 30 & $56 \pm 7$ & $11 \pm 3$ \\
G29.96 & $45 \pm 9$ & $48 \pm 8$ & $4 \pm 1$ \\
G31.41 & $32 \pm 8$ & $53 \pm 8$ & $15 \pm 2$ \\
& $29 \pm 5\left(^{* *}\right)$ & $51 \pm 7$ & $15 \pm 3$ \\
& $26 \pm 5\left(^{* * *}\right)$ & $48 \pm 7$ & $16 \pm 3$ \\
G34.26 & $40 \pm 9$ & $46 \pm 9$ & $16 \pm 2$ \\
G45.47 & $35 \pm 8$ & $39 \pm 7$ & $5.0 \pm 0.3$ \\
W51D $\left(^{*}\right)$ & 23 & $47 \pm 10$ & $15 \pm 3$ \\
IRAS 20126 & $22 \pm 7$ & $30 \pm 5$ & $3.9 \pm 0.2$ \\
\hline
\end{tabular}

$\left.{ }^{*}\right)$ Sources for which the angular diameter could not be computed. For G10.30 and W51D $T_{\text {rot }}$ and $N_{\text {tot }}$ have been computed assuming a linear diameter equal to the mean value (0.9 pc) obtained from the other sources. For G19.61, instead, we have assumed the diameter of $30^{\prime \prime}$ derived by Hofner et al. (2000) from the $\mathrm{C}^{17} \mathrm{O}$ emission.

$\left({ }^{* *}\right)$ Angular diameter measured from the $\mathrm{CH}_{3} \mathrm{C}_{2} \mathrm{H}(6-5)$ map of Fig. 2.

$\left({ }^{* * *}\right)$ Angular diameter measured from the $\mathrm{CH}_{3} \mathrm{C}_{2} \mathrm{H}(8-7)$ map of Fig. 3.

next section that the molecular hydrogen densities turn out to be greater than this value, which supports our assumption of LTE (and hence $T_{\text {rot }} \simeq T_{\mathrm{k}}$ ) for the $\mathrm{CH}_{3} \mathrm{C}_{2} \mathrm{H}$ molecules.

As for the optically thin assumption, it is worth noting that the observed bandwidth covers most of the $K$ components of the $\mathrm{CH}_{3}{ }^{13} \mathrm{CCH}(6-5)$, (8-7), and (13-12) transitions. One can thus estimate the optical depth from the intensity ratio between the lines of the main species and those of the isotopomer. However, none of the $\mathrm{CH}_{3}{ }^{13} \mathrm{CCH}$ lines is detected by us, so that only an upper limit on the optical depth can be obtained: assuming an isotopic ratio ${ }^{12} \mathrm{C} /{ }^{13} \mathrm{C}=50$ (see Wilson \& Rood 1994), the minimum value turns out to be $\tau=8$, which is insufficient to demonstrate that the $\mathrm{CH}_{3} \mathrm{C}_{2} \mathrm{H}$ lines are optically thin. Nevertheless, the excellent agreement between the linear fit and the data in the Boltzmann plots reinforces the assumption of optically thin line emission: in fact, large optical depths are expected to affect mostly the low excitation transitions and hence cause a flattening of the plots at low energies, which is not seen in Fig. 4.

We will examine the optical depth issue in some better detail in Sect. 3.4.1.

Another topic which may be discussed in relation to the fits in Fig. 4, is the effect of temperature and density gradients on the rotation diagrams: we delay this to Sects. 3.4.2 and 3.4.3.

\subsection{Mass and density}

By means of the parameters derived above, we can estimate the mass of the clumps and the corresponding virial mass needed for equilibrium. For both estimates, spherical symmetry is assumed.

The mass of the clump, $M_{\mathrm{CD}}$, can be obtained from the column density in Table 7: for a spherical, isothermal clump one has

$M_{\mathrm{CD}}=\frac{\pi}{4} \frac{\Theta_{\mathrm{s}}^{2} d^{2} N_{\mathrm{tot}} m_{\mathrm{H}_{2}}}{X_{\mathrm{CH}_{3} \mathrm{C}_{2} \mathrm{H}}}$

where $d$ is the distance to the source, $m_{\mathrm{H}_{2}}$ the mass of the $\mathrm{H}_{2}$ molecule, $X_{\mathrm{CH}_{3} \mathrm{C}_{2} \mathrm{H}}$ the abundance of $\mathrm{CH}_{3} \mathrm{C}_{2} \mathrm{H}$ relative to $\mathrm{H}_{2}$, and $N_{\text {tot }}$ is the mean column density over the clump, as previously explained.

The most uncertain value in Eq. (5) is $X_{\mathrm{CH}_{3} \mathrm{C}_{2} \mathrm{H}}$. Rather than assuming a priori an arbitrary value for a parameter which is known to vary by a large amount in the interstellar medium, we have decided to obtain an estimate of it by equating Eq. (5) to the mass, $M_{\text {cont }}$, derived from continuum measurements of our sources (the latter is given in Table 8). To this purpose, we used the (sub) millimeter continuum emission observations made by various authors (Chini et al. 1986; Cesaroni et al. 1999; Hatchell et al. 2000; Hunter et al. 2000) towards most of our sources. Following Mezger et al. (1990), the mass was derived using these continuum measurements and the dust temperatures quoted by the corresponding authors; when the latter were not available, we used $T_{\text {rot }}$ from Table 7 . The values of $X_{\mathrm{CH}_{3} \mathrm{C}_{2} \mathrm{H}}$ obtained by us range from $0.9 \times 10^{-9}$ to $5.7 \times 10^{-9}$ (see Table 8 ) with a mean value of $\sim 2.2 \times 10^{-9}$. We have hence assumed $X_{\mathrm{CH}_{3} \mathrm{C}_{2} \mathrm{H}}=2 \times 10^{-9}$, with an uncertainty of a factor $\sim 2.5$ and computed the $M_{\mathrm{CD}}$ from Eq. (5) using this value for all sources. The results are given in Table 9 .

It is worth pointing out that the continuum measurements do not necessarily refer to the same region seen in the $\mathrm{CH}_{3} \mathrm{C}_{2} \mathrm{H}$ lines: however, in a few cases (G5.89, G9.62, G10.47, G31.41) maps in the (sub)millimeter continuum are available, so that we could verify the coincidence between the $\mathrm{CH}_{3} \mathrm{C}_{2} \mathrm{H}$ and dust emitting regions $\left(\sim 30^{\prime \prime}\right)$. For the remaining sources, the continuum observations were performed with an instrumental HPBW greater than ours, so that the mass estimates in Table 8 are to be taken as upper limits to the mass of the clumps traced by $\mathrm{CH}_{3} \mathrm{C}_{2} \mathrm{H}$, which in turn imply lower limits on the $\mathrm{CH}_{3} \mathrm{C}_{2} \mathrm{H}$ abundances; however, the relatively small range of variation of $X_{\mathrm{CH}_{3} \mathrm{C}_{2} \mathrm{H}}$ in Table 8 suggests that our assumption of $X_{\mathrm{CH}_{3} \mathrm{C}_{2} \mathrm{H}}=2 \times 10^{-9}$ is likely to be a reliable approximation.

The virial mass for a homogeneous spherical clump, neglecting contributions from magnetic field and surface pressure, can be computed from the expression (see e.g. MacLaren et al. 1988)

$M_{\text {vir }}\left(M_{\odot}\right)=0.509 d(\mathrm{kpc}) \Theta_{\mathrm{s}}(\operatorname{arcsec}) \Delta v_{1 / 2}^{2}\left(\mathrm{~km} \mathrm{~s}^{-1}\right)$

where $\Delta v_{1 / 2}$ is the observed line FWHM. In our case we took $\Delta v_{1 / 2}$ from the $\mathrm{CH}_{3} \mathrm{C}_{2} \mathrm{H}(6-5)$ lines, for the sake 
of consistency with the derivation of $\Theta_{\mathrm{s}}$ which has also been obtained from the $\mathrm{CH}_{3} \mathrm{C}_{2} \mathrm{H}(6-5)$ emission. The values of $M_{\text {vir }}$ are given in Table 9 .

In the same table we also give the $\mathrm{H}_{2}$ volume densities estimated from $M_{\mathrm{CD}}$ and the clump diameters, $D$. The uncertainties on these values have been calculated by means of the propagation of statistical errors, but they do not take into account distance uncertainties. All masses are equal to a few $\sim 10^{3} M_{\odot}$, with the sole exception of IRAS 20126, for which the mass is $\lesssim 10^{2} M_{\odot}$. The $\mathrm{H}_{2}$ volume densities range from $10^{5}$ to $5 \times 10^{6} \mathrm{~cm}^{-3}$, greater than the maximum critical density of the transitions observed (see the discussion in Sect. 3.1).

\subsection{Effects of inhomogeneity and optical depth}

In view of the findings that will be illustrated in Sect. 4, before proceeding further it is important to discuss the relevance of optical depth effects, temperature gradients, and density gradients on the mass (and hence density) estimates obtained so far.

\subsubsection{Optical depth}

As explained in Sect. 3.2, we believe that the bulk of the observed $\mathrm{CH}_{3} \mathrm{C}_{2} \mathrm{H}$ emission is optically thin. However, one cannot exclude the existence of an optically thick region close to the centre of the clump, where the density is presumably higher than average. Indeed, dense molecular cores with diameters of $\sim 0.1 \mathrm{pc}$ are known to exist in most of our clumps. Hence the question is how much of the clump mass is contained in the optically thick region. One can calculate the size of the region over which $\tau \geq 1$ for the $\mathrm{CH}_{3} \mathrm{C}_{2} \mathrm{H}$ lines. For $N_{\text {tot }}=2 \times 10^{15} \mathrm{~cm}^{-2}, T=40 \mathrm{~K}$, $\Delta v_{1 / 2}=5 \mathrm{~km} \mathrm{~s}^{-1}$, and a density profile $n \propto R^{-2}$ one finds that the optical depth of e.g. the $\mathrm{CH}_{3} \mathrm{C}_{2} \mathrm{H}(6-5) K=0$ transition along the line of sight passing through the centre of the clump is equal to $\tau \simeq 0.1\left(R_{0} / R-1\right)$, where $R_{0}$ is the radius of the clump and $R$ the distance from the centre at which the optical depth $\tau$ is achieved. By posing $\tau=1$ one finds the radius of the optically thick core, $R / R_{0} \simeq 10$. In conclusion, the $\mathrm{CH}_{3} \mathrm{C}_{2} \mathrm{H}$ gas becomes optically thick at a distance from the centre equal to $\sim 10 \%$ of the clump radius: this contains $\sim 10 \%$ of the total mass. We conclude that optical depth effects are negligible for the estimate of $M_{\mathrm{CD}}$.

Incidentally, it is worth noting that in case of large optical depths the values of $N_{\text {tot }}$ and $M_{\mathrm{CD}}$ are to be taken as lower limits. On the contrary, $M_{\text {vir }}$ becomes an upper limit, because for $\tau \gg 1$ line broadening occurs, which increases the value of $\Delta v_{1 / 2}$ used in Eq. (6). As a consequence, the ratio $M_{\mathrm{CD}} / M_{\mathrm{vir}}$ which one can derive from Table 9 has to be regarded as a lower limit. This fact reinforces the conclusion attained later in Sect. 4.1.

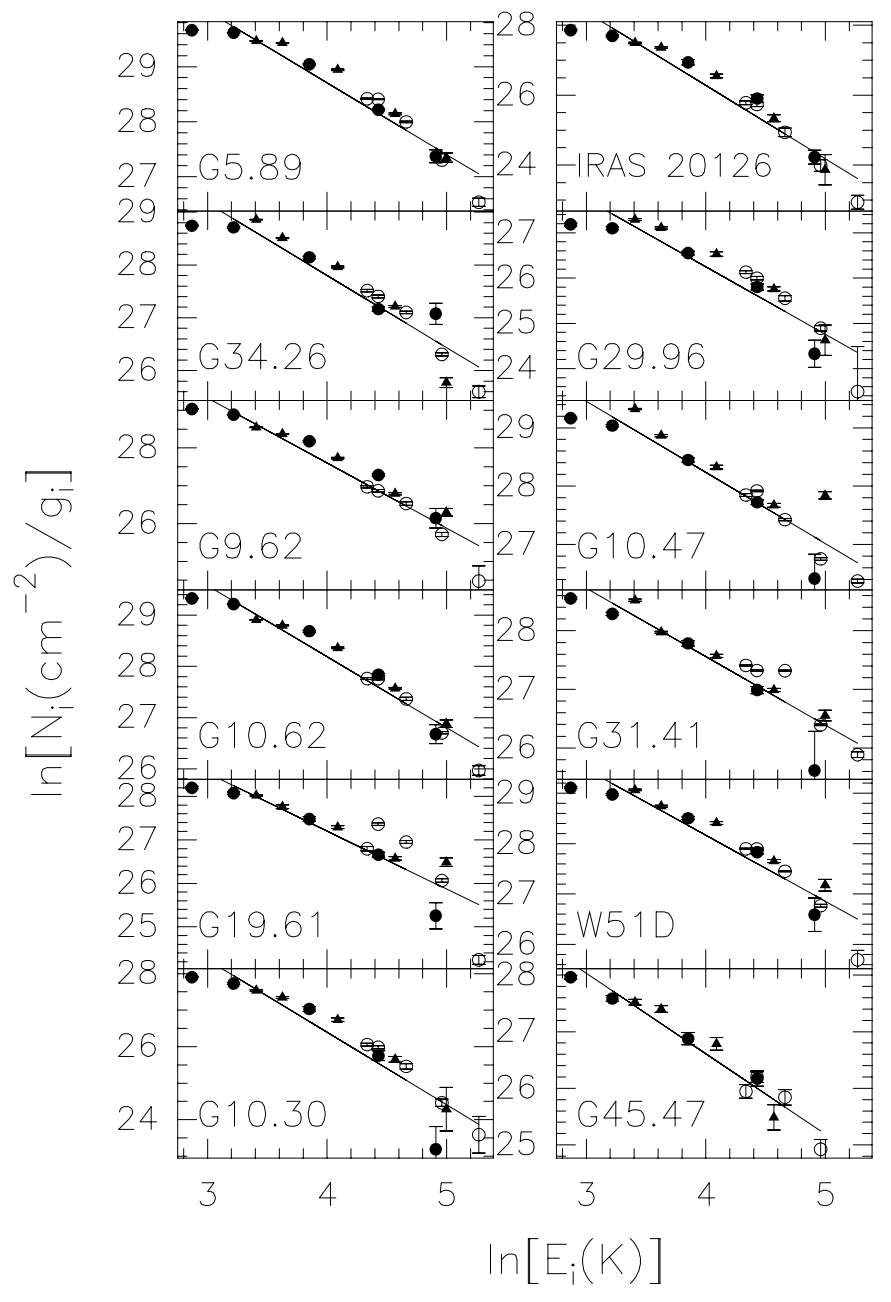

Fig. 5. Same as Fig. 4 but with logarithmic scale on the abscissa.

\subsubsection{Temperature gradient}

The hypotheses under which the method of the population diagrams in Sect. 3.2 is applied are that the gas is optically thin, in LTE, and isothermal. The first two assumptions have already been discussed; as for the latter, Mauersberger et al. (1988) have treated the case of a spherically symmetric cloud with temperature and density having power-law dependence on the distance from the centre. These authors demonstrate that in this case a linear correlation should hold between $\ln N_{\mathrm{i}}$ and $\ln E_{\mathrm{i}}$, unlike the isothermal case where the correlation is expected with $E_{\mathrm{i}}$. We stress that the different dependence on $E_{\mathrm{i}}$ is caused only by the temperature gradient: in fact, for an isothermal cloud the usual linear relation between $\ln N_{\mathrm{i}}$ and $E_{\mathrm{i}}$ is recovered, no matter what the density gradient is.

Following the example of Mauersberger et al. (1988), in Fig. 5 we present "modified" population diagrams for our sources, plotting $\ln N_{\mathrm{i}}$ versus $\ln E_{\mathrm{i}}$. Clearly, the linear fits to the points are much less satisfactory than those in Fig. 4. We conclude that the $\mathrm{CH}_{3} \mathrm{C}_{2} \mathrm{H}$ emission arises from a region in which the temperature profile must 
Table 8. Mass and $\mathrm{CH}_{3} \mathrm{C}_{2} \mathrm{H}$ abundance estimates from the continuum millimeter emission.

\begin{tabular}{lcccc}
\hline \hline Source & $\begin{array}{c}d^{(a)} \\
(\mathrm{kpc})\end{array}$ & $\begin{array}{c}M_{\text {cont }} \\
\left(M_{\odot}\right)\end{array}$ & $\begin{array}{c}X_{\mathrm{CH}_{3} \mathrm{C}_{2} \mathrm{H}} \\
\left(\times 10^{-9}\right)\end{array}$ & Ref. \\
\hline G5.89 & 4.0 & 1500 & 5.5 & 1 \\
G9.62 & 5.7 & 1600 & 5.7 & 1 \\
G10.47 & 5.8 & 12700 & 0.9 & 2 \\
G10.30 & 6.0 & - & - & - \\
G10.62 & 6.0 & 8700 & 1.7 & 3 \\
G19.61 & 3.5 & 2200 & 1.4 & 3 \\
G29.96 & 7.4 & 11200 & 1.0 & \\
G31.41 & 7.9 & 7300 & 3.5 & 2 \\
G34.26 & 4.0 & 9700 & 1.1 & 3 \\
G45.47 & 8.3 & 12900 & 0.9 & 3 \\
W51D & 8.0 & - & - & - \\
IRAS 20126 & 1.7 & 110 & 1.3 & 4 \\
\hline
\end{tabular}

Reference codes: $1=$ Hunter et al. (2000); $2=$ Hatchell et al. (2000); $3=$ Chini et al. (1987); $4=$ Cesaroni et al. (1999).

(a) Distances are from Wood \& Churchwell (1989a) for G10.30 and from Kurtz et al. (2000) for the other sources.

be very shallow, hence making $T=$ const. a suitable approximation.

\subsubsection{Density gradient}

For an isothermal and optically thin cloud, the source averaged column density cannot depend on the way the gas is distributed inside the cloud: hence, density gradients leave the estimates of $N_{\text {tot }}$ and $M_{\mathrm{CD}}$ unaffected. On the other hand, the virial mass depends on the density profile, as demonstrated e.g. by MacLaren et al. (1988). For a power-law density distribution of the type $n_{\mathrm{H}_{2}} \propto R^{p}$, the virial mass obtained from Eq. (6) must be multiplied by the factor $\frac{3}{5} \frac{5+2 p}{3+p}$, which is $\leq 1$ for $p \leq 0$. Therefore, the values of $M_{\text {vir }}$ of Table 9 derived for a homogeneous cloud are to be taken as upper limits.

In conclusion, the ratio $M_{\mathrm{CD}} / M_{\mathrm{vir}}$ derived by us represents an underestimate if the constant density assumption is released in the computation of $M_{\mathrm{vir}}$. This result is important in view of the discussion in Sect. 4.1.

\section{Discussion}

In the previous section, we have demonstrated that the values of the physical parameters derived by us are robust. In particular, we have shown that: (i) optical depth effects can be neglected for the $\mathrm{CH}_{3} \mathrm{C}_{2} \mathrm{H}$ lines; (ii) temperature gradients, if present, must be very "flat" in the $\mathrm{CH}_{3} \mathrm{C}_{2} \mathrm{H}$ emitting region; and (iii) density gradients have no effect on the estimate of $M_{\mathrm{CD}}$ and the corresponding $\mathrm{H}_{2}$ density. In the following, we shall use these physical parameters to analyse the stability and structure of the clumps.

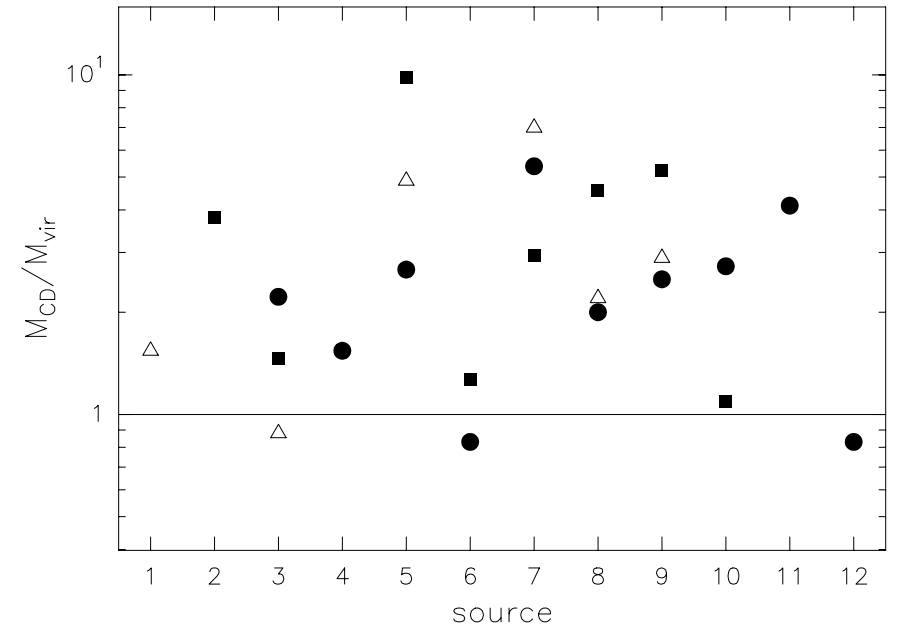

Fig. 6. Plot of the ratio between the mass estimated from the gas column density and that derived assuming virial equilibrium for the clumps observed in this study. Full circles indicate values computed from $\mathrm{CH}_{3} \mathrm{C}_{2} \mathrm{H}$ (this study), empty triangles those derived from $\mathrm{C}^{34} \mathrm{~S}$ (Cesaroni et al. 1991), and filled squares those obtained from $\mathrm{C}^{17} \mathrm{O}$ (Hofner et al. 2000). The straight line corresponds to $M_{\mathrm{CD}}=M_{\mathrm{vir}}$.

\subsection{Stability of the clumps}

From Table 9 one can see that the mean ratio between $M_{\mathrm{CD}}$ and $M_{\text {vir }}$ is $\sim 3.0 \pm 1.7$, being $\sim 1$ only in the cases of G19.61 and IRAS 20126. Although such a ratio is only marginally $>1$, it is such for all of the sources but two of them: this result seems too systematic to be due only to random errors on the quantities of interest. The question is whether the ratio $M_{\mathrm{CD}} / M_{\mathrm{vir}}$ can be reduced to unity by any means. Let us examine a few possibilities.

As already discussed in Sects. 3.4.1 and 3.4.3, optical depth effects and density gradients can only increase the ratio $M_{\mathrm{CD}} / M_{\mathrm{vir}}$, as they make $M_{\mathrm{CD}}$ bigger and $M_{\mathrm{vir}}$ smaller.

Another possibility is that the $\mathrm{CH}_{3} \mathrm{C}_{2} \mathrm{H}$ abundance is underestimated, which would cause an overestimate of $M_{\mathrm{CD}}$. In principle this cannot be excluded, given the large variations of molecular abundances in different objects (see e.g. Irvine et al. 1987); however, our estimate of $X_{\mathrm{CH}_{3} \mathrm{C}_{2} \mathrm{H}}$ (see Sect. 3.3) is "direct", as it makes use of the continuum emission from the regions of interest and it is hence less prone to errors which instead affect values based on chemical models. Further evidence for $M_{\mathrm{CD}}$ being greater than $M_{\text {vir }}$ comes from the studies of Cesaroni et al. (1991) and Hofner et al. (2000) who mapped some of our clumps respectively in the $\mathrm{C}^{34} \mathrm{~S}$ and $\mathrm{C}^{17} \mathrm{O}$ lines: as one can see in Fig. 6 , in all sources the ratio $M_{\mathrm{CD}} / M_{\text {vir }}$ is basically $>1$, independently of the tracer. It is worth stressing that the values of $M_{\mathrm{CD}}$ obtained by Hofner et al. (2000) from $\mathrm{C}^{17} \mathrm{O}$ are less affected by uncertainties on the abundance, which is much better established for $\mathrm{C}^{17} \mathrm{O}$ than for $\mathrm{CH}_{3} \mathrm{C}_{2} \mathrm{H}$.

In conclusion, we favour the hypothesis that the discrepancy between $M_{\mathrm{CD}}$ and $M_{\mathrm{vir}}$ is real. In this case 


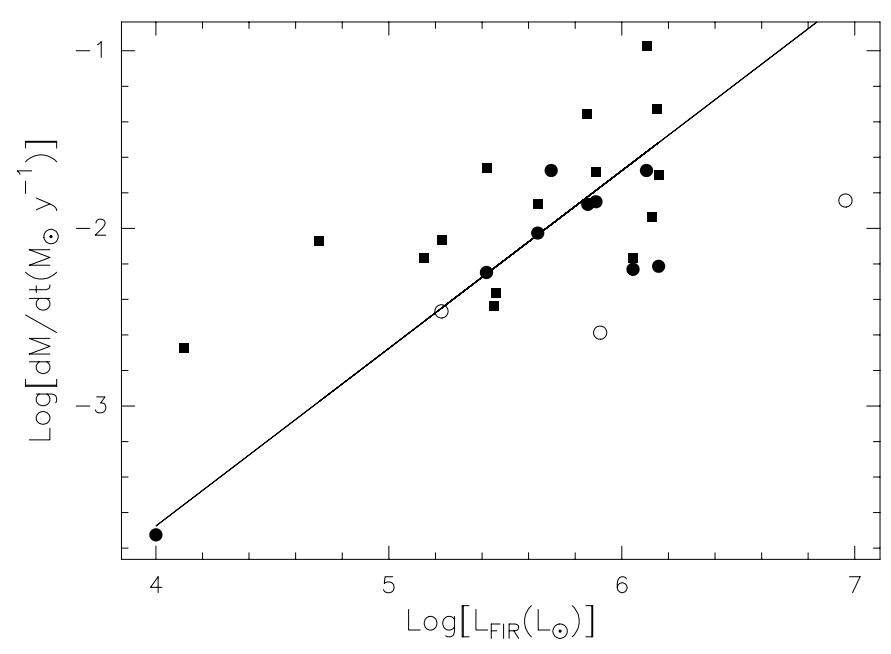

Fig. 7. Plot of the mass accretion rate versus the source bolometric luminosity estimated from the IRAS fluxes. Circles and squares indicate estimates obtained respectively from $\mathrm{CH}_{3} \mathrm{C}_{2} \mathrm{H}$ (this study) and $\mathrm{C}^{17} \mathrm{O}$ (Hofner et al. 2000). Empty circles indicate the sources for which it was not possible to estimate the size of the $\mathrm{CH}_{3} \mathrm{C}_{2} \mathrm{H}$ clump (see Table 7). The straight line is the best fit assuming proportionality between mass accretion rate and luminosity and corresponds to $\dot{M}_{\text {acc }}\left(M_{\odot \mathrm{yr}^{-1}}\right)=$ $2.1 \times 10^{-8} L_{\mathrm{FIR}}\left(L_{\odot}\right)$.

$M_{\mathrm{CD}} / M_{\mathrm{vir}}>1$ seems to indicate that the clumps are unstable against gravitational collapse.

If thermal pressure and turbulence are the only means of support against gravitational collapse, indeed $M_{\mathrm{CD}}>$ $M_{\mathrm{vir}}$ implies collapse of the clumps on a free-fall time: for densities of order $10^{6} \mathrm{~cm}^{-3}$ the latter is equal to $\sim 10^{4} \mathrm{yr}$, an order of magnitude less than the estimated lifetime of the UC Hir regions embedded in the clumps (Wood \& Churchwell 1989b). This suggests that magnetic fields might play a role in stabilising the cloud against gravitational collapse. From the virial theorem one can estimate the strength of the magnetic field, $B$, required to this purpose: following McKee et al. (1993) and neglecting the surface pressure term, the expression for virial equilibrium can be written as

$3 M \frac{\Delta v_{1 / 2}^{2}}{8 \ln 2}+\frac{b}{3} B^{2} R^{3}=\frac{3 a}{5} \frac{G M^{2}}{R}$

where $a$ and $b$ are two factors of order unity that take into account the cloud geometry and the topology of the magnetic field. The equilibrium value of $B^{2}$ is hence given by

$B^{2}=\frac{9}{5} \frac{a}{b} \frac{G M}{R^{4}}\left(M-\frac{5}{8 \ln 2} \frac{R \Delta v_{1 / 2}^{2}}{a G}\right)$.

Assuming $a=1.17$ and $b=0.3$ (McKee et al. 1993) and posing $M=M_{\mathrm{CD}}$ we can compute the values of $B$ required to support our clumps: these are a few $\mathrm{mG}$. Such values are comparable to those obtained by Lai et al. (2001) who measured the magnetic field in molecular clouds with densities similar to those of our clumps using interferometric polarisation maps.
We thus propose a scenario in which the clumps are marginally stable due to the contribution of the magnetic field. In this case the clump will contract on the timescale $t_{\mathrm{AD}}$ determined by ambipolar diffusion. This can be related to the free-fall time, $t_{\mathrm{ff}}$, by means of Eq. (51) of McKee et al. (1993): $t_{\mathrm{AD}} \simeq 8.5 t_{\mathrm{ff}}$, thus obtaining $t_{\mathrm{AD}}(\mathrm{yr})=2.9 \times 10^{8}\left[n_{\mathrm{H}_{2}}\left(\mathrm{~cm}^{-3}\right)\right]^{-0.5}$. The mass accretion rate onto the embedded stars can be hence computed from the ratio $\dot{M}_{\text {acc }}=M_{\mathrm{CD}} / t_{\mathrm{AD}}$ and plotted as a function of the source luminosity, as done in Fig. 7: in this figure we have also reported the estimates obtained from the $\mathrm{C}^{17} \mathrm{O}$ data of Hofner et al. (2000). The accretion rate increases with source luminosity: this is consistent with the finding that the star formation rate in a cloud is roughly proportional to the bolometric luminosity according to the expression $\dot{M}_{*}\left(M_{\odot} \mathrm{yr}^{-1}\right) \simeq 4 \times 10^{-10} L\left(L_{\odot}\right)$ (Plume et al. 1997). Therefore, it is reasonable to expect that also $\dot{M}_{\text {acc }}$ satisfies a similar relationship. We hence fitted the data in Fig. 7 assuming $\dot{M}_{\text {acc }} \propto L_{\mathrm{FIR}}$, thus obtaining $\dot{M}_{\text {acc }} / L_{\text {FIR }}=2.1 \times 10^{-8} M_{\odot} \mathrm{yr}^{-1} L_{\odot}^{-1}$. The ratio $\dot{M}_{*} / \dot{M}_{\text {acc }} \simeq 1.9 \%$ is the star formation efficiency in the clumps. On the other hand, a lower limit to the same value can be obtained from the ratio between the mass of the early-type star ionising the embedded UC HiI region and the mass of the corresponding clump: this is approximately $30 M_{\odot} / 5000 M_{\odot} \simeq 0.6 \%$, consistent with the previous estimate. It is also worth noting that the mass accretion rates are of the order of $\sim 10^{-2} M_{\odot} \mathrm{yr}^{-1}$ : values such high could support theories which predict that high-mass stars can form through accretion (Behrend \& Maeder 2001).

In conclusion, although other explanations are possible, we cannot exclude the possibility that the molecular clumps traced by the $\mathrm{CH}_{3} \mathrm{C}_{2} \mathrm{H}$ transitions are on the edge of gravitational collapse. In support of this hypothesis we note that evidence for infall in some of our clumps has been found by other authors on the basis of high angular resolution observations in various molecular tracers (Hofner et al. 1999; Maxia et al. 2001). In Sect. 4.2.2 we will demonstrate that also the density structure of the clumps favours the scenario presented above.

\subsection{Internal structure of the clumps}

We have found that the clumps seen in the $\mathrm{CH}_{3} \mathrm{C}_{2} \mathrm{H}$ lines have mean diameters of $0.9 \mathrm{pc}$, kinetic temperatures of $\sim 40 \mathrm{~K}$ and $\mathrm{H}_{2}$ volume densities of $10^{5}-10^{6} \mathrm{~cm}^{-3}$. It is interesting to compare these values with those derived from other tracers.

For the same quantities, Hofner et al. (2000) find slightly different mean values from their $\mathrm{C}^{17} \mathrm{O}$ observations, namely diameters of $\sim 1.1 \mathrm{pc}$, kinetic temperatures of $\sim 20 \mathrm{~K}$ and $\mathrm{H}_{2}$ volume densities of $\sim 10^{4} \mathrm{~cm}^{-3}$. This means that the $\mathrm{C}^{17} \mathrm{O}$ emission arises from larger, colder, and less dense regions than $\mathrm{CH}_{3} \mathrm{C}_{2} \mathrm{H}$. This is not surprising as the $\mathrm{C}^{17} \mathrm{O}$ is an order of magnitude more abundant than $\mathrm{CH}_{3} \mathrm{C}_{2} \mathrm{H}$ and hence likely to trace lower density gas. 
Table 9. Mass and $\mathrm{H}_{2}$ density estimates.

\begin{tabular}{lcccc}
\hline \hline Source & $\begin{array}{c}D \\
(\mathrm{pc})\end{array}$ & $\begin{array}{c}M_{\text {vir }} \\
\left(M_{\odot}\right)\end{array}$ & $\begin{array}{c}M_{\mathrm{CD}}^{(a)} \\
\left(M_{\odot}\right)\end{array}$ & $\begin{array}{c}n_{\mathrm{H}_{2}}^{\mathrm{CD}} \\
\left(10^{5} \mathrm{~cm}^{-3}\right)\end{array}$ \\
\hline G5.89 & $0.6 \pm 0.2$ & $1800 \pm 300$ & $4500 \pm 1200$ & $8 \pm 2$ \\
G9.62 & $0.8 \pm 0.2$ & $1000 \pm 400$ & $5000 \pm 1000$ & $5 \pm 1$ \\
G10.47 & $0.6 \pm 0.1$ & $2700 \pm 1000$ & $6000 \pm 2000$ & $10 \pm 3$ \\
G10.30 & 0.8 & $1300 \pm 400$ & $2000 \pm 1000$ & $20 \pm 10$ \\
G10.62 & $0.8 \pm 0.2$ & $3000 \pm 1000$ & $8000 \pm 2000$ & $2.5 \pm 0.8$ \\
G19.61 & 0.5 & $1800 \pm 500$ & $1500 \pm 500$ & $7 \pm 2$ \\
G29.96 & $1.6 \pm 0.3$ & $1300 \pm 400$ & $7000 \pm 2000$ & $0.7 \pm 0.2$ \\
G31.41 & $1.2 \pm 0.3$ & $2500 \pm 600$ & $5000 \pm 2000$ & $3 \pm 1$ \\
G34.26 & $0.8 \pm 0.2$ & $2400 \pm 700$ & $6000 \pm 1500$ & $6 \pm 2$ \\
G45.47 & $1.4 \pm 0.3$ & $2200 \pm 800$ & $6000 \pm 2000$ & $0.9 \pm 0.3$ \\
W51D & 0.9 & $1700 \pm 600$ & $7000 \pm 3000$ & $50 \pm 20$ \\
IRAS 20126 & $0.18 \pm 0.06$ & $90 \pm 30$ & $75 \pm 20$ & $5 \pm 1$ \\
\hline
\end{tabular}

(a) Calculated assuming a $\mathrm{CH}_{3} \mathrm{C}_{2} \mathrm{H}$ abundance of $2 \times 10^{-9}$.

On the other hand, the $\mathrm{C}^{34} \mathrm{~S}$ observations of Cesaroni et al. (1991) and Olmi \& Cesaroni (1999) provide greater densities and smaller diameters than our $\mathrm{CH}_{3} \mathrm{C}_{2} \mathrm{H}$ measurements, namely $\sim 10^{6} \mathrm{~cm}^{-3}$ and $\sim 0.4 \mathrm{pc}$.

Finally, Kurtz et al. (2000) compiled a list of the properties of hot molecular cores (see their Table 1): such cores are present in most of our sources and represent the innermost, densest parts of the clumps seen in $\mathrm{CH}_{3} \mathrm{C}_{2} \mathrm{H}$, with typical temperatures of $\sim 100 \mathrm{~K}$ and diameters $\lesssim 0.1 \mathrm{pc}$.

In the following we make use of all these information to derive the distribution of the physical parameters in the clumps.

\subsubsection{Temperature structure}

If we assume that the gas of the clump is heated by an embedded high-mass star, the gas temperature, $T$, at a distance $R=\theta d$ from the star is given by the expression (see e.g. Plume et al. 1997 and references therein)

$T=C\left(\frac{L^{\frac{1}{2}}}{\theta d}\right)^{-q}$

with $d$ distance to the source, $L$ luminosity of the embedded star, and $\theta$ angular distance from the star. In order to verify whether such a relation holds for our clumps and to compute the values of $C$ and $q$, in Fig. 8 we plot $\log (T)$ versus $\log \left[L_{\mathrm{FIR}}^{1 / 2} /(\theta d)\right]$ : note that both quantities are distance independent. The distribution can be fitted with a straight line corresponding to $q=-0.54 \pm 0.06$ and $C=10 \pm 2$, when $T$ is expressed in $\mathrm{K}, L_{\mathrm{FIR}}$ in $L_{\odot}, d$ in $\mathrm{kpc}$, and $\theta$ in arcsec.

Equation (9) implies that for a given source $T \propto \theta^{q}$. Therefore, one can also derive $q$ from the ratio between the temperatures measured in two different tracers and the

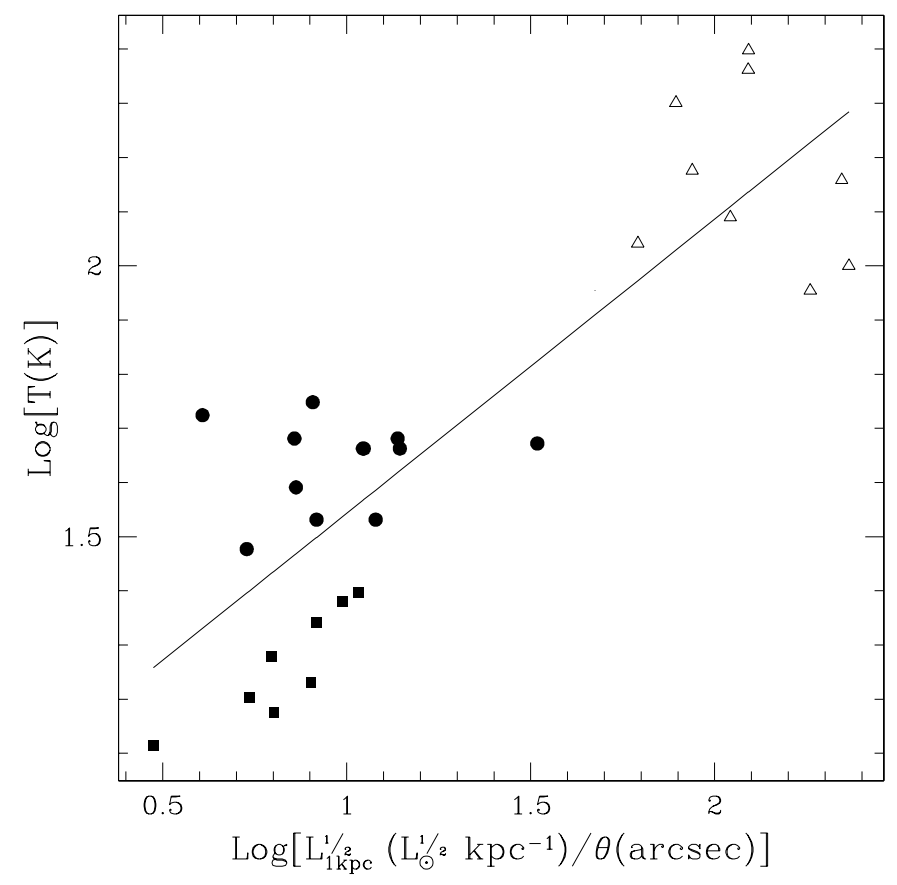

Fig. 8. Plot of the kinetic temperature versus the quantity $L_{1 \mathrm{kpc}} / \theta$, where $L_{1 \mathrm{kpc}}$ is the source luminosity scaled to the distance of $1 \mathrm{kpc}$ and $\theta$ the angular radius at which the temperature has been measured. Filled circles and squares indicate respectively estimates based on $\mathrm{CH}_{3} \mathrm{C}_{2} \mathrm{H}$ (this study) and $\mathrm{C}^{17} \mathrm{O}$ (Hofner et al. 2000), while empty triangles correspond to the values of Table 1 of Kurtz et al. (2000). The straight line represents a least square fit to the data, with slope $0.54 \pm 0.06$ and intercepta $1 \pm 0.09$.

ratio between the corresponding diameters, which satisfy the relation

$\frac{T_{X}}{T_{Y}}=\left(\frac{R_{X}}{R_{Y}}\right)^{q}$ 


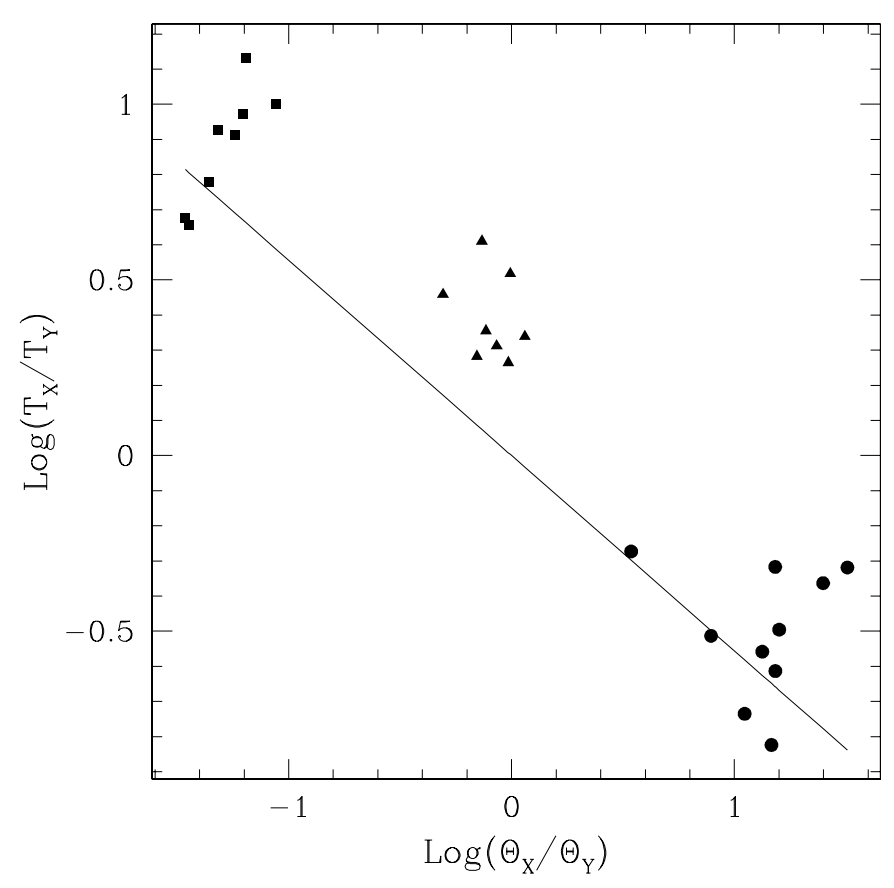

Fig. 9. Plot of the temperature ratio $T_{X} / T_{Y}$ versus the angular diameter ratio $\Theta_{X} / \Theta_{Y}$, where $X$ and $Y$ indicate two estimates among those derived from $\mathrm{CH}_{3} \mathrm{C}_{2} \mathrm{H}, \mathrm{C}^{17} \mathrm{O}$ (Hofner et al. 2000), and those of Table 1 of Kurtz et al. (2000). Circles correspond to ratios between the temperatures quoted by Kurtz et al. and those obtained from $\mathrm{CH}_{3} \mathrm{C}_{2} \mathrm{H}$, squares to ratios between estimates from Kurtz et al. and from $\mathrm{C}^{17} \mathrm{O}$, and triangles to ratios between estimates from $\mathrm{CH}_{3} \mathrm{C}_{2} \mathrm{H}$ and $\mathrm{C}^{17} \mathrm{O}$. The straight line represents a least square fit of the type $T \propto \theta^{q}$, with $q=-0.56 \pm 0.06$. The correlation coefficient is -0.93 .

with $X, Y$ indicating that the value has been obtained from $\mathrm{CH}_{3} \mathrm{C}_{2} \mathrm{H}, \mathrm{C}^{17} \mathrm{O}$ (Hofner et al. 2000), or taken from Table 1 of Kurtz et al. (2000). Under the assumption that $q$ is exactly the same for all sources, Eq. (10) is totally source independent and one can plot all possible ratios for all sources in the same figure. The advantage of this approach with respect to that previously used is that we do not need any luminosity estimate. The result is shown in Fig. 9: the slope in this case is $q=-0.56 \pm 0.06$, consistent with the previous estimate.

We conclude that the temperature in the clumps is approximately described by the law $T \propto R^{-0.55}$. Such a law is steeper than expected on the basis of the discussion of Sect. 3.4.2, where we conclude that no temperature gradient is seen in the gas traced by the $\mathrm{CH}_{3} \mathrm{C}_{2} \mathrm{H}$ emission. However, in order to reveal this gradient we had to compare the temperature measured on the large scale (in the $\mathrm{CH}_{3} \mathrm{C}_{2} \mathrm{H}$ and $\mathrm{C}^{17} \mathrm{O}$ emission) with that on a very small scale (in $\mathrm{CH}_{3} \mathrm{CN}$ ). This means that while $T \propto R^{-0.55}$ is a reasonable description of the "global" temperature variation from $\sim 0.1 \mathrm{pc}$ to $\sim 1 \mathrm{pc}$, it cannot be applied rigorously at all radii in the clump. In particular, the temperature profile is likely to be shallow in the outer region (for the reason discussed in Sect. 3.4.2) and steeper close to the center.
Note also that $q=-0.55$ is significantly greater than the slope expected for an optically thin clump heated by an embedded energy source. In fact, in this case for a dust absorption coefficient $\propto \nu^{\beta}$ one finds $T \propto R^{-2 /(4+\beta)}$ : the steepest slope is obtained for $\beta=1$ and is equal to -0.4 . A plausible explanation for $q$ being less than -0.4 is that part of the clump is optically thick. Indeed, in some of our objects, on scales 10 times smaller than the clumps, Cesaroni et al. (1998) found optically thick, hot cores with temperature profiles $T \propto R^{-0.75}$. These cores are just the regions which the temperature estimates by Kurtz et al. (2000) used in our plots refer to. We thus conclude that one may read the slope derived by us as a "compromise" between a flatter temperature profile in the optically thin clump - traced by $\mathrm{C}^{17} \mathrm{O}$ and $\mathrm{CH}_{3} \mathrm{C}_{2} \mathrm{H}$ - and the steeper profile $\left(T \propto R^{-0.75}\right)$ in the hot core-measured e.g. in $\mathrm{NH}_{3}$ or $\mathrm{CH}_{3} \mathrm{CN}$.

\subsubsection{Density structure}

For star forming clumps, theoretical models predict density profiles of the type $n \propto R^{p}$. In our case, it is difficult to test this prediction source by source, as only few measurements for each source at different radii are available, namely those from $\mathrm{CH}_{3} \mathrm{C}_{2} \mathrm{H}, \mathrm{C}^{34} \mathrm{~S}, \mathrm{C}^{17} \mathrm{O}$, and the density estimates obtained from Table 1 of Kurtz et al. (2000). However, the ratio between the $\mathrm{H}_{2}$ densities measured with different tracers is equal to

$\frac{n_{X}}{n_{Y}}=\left(\frac{R_{X}}{R_{Y}}\right)^{p}$

with $n_{X, Y}$ density measured with tracer $X, Y=\mathrm{CH}_{3} \mathrm{C}_{2} \mathrm{H}$, $\mathrm{C}^{17} \mathrm{O}, \mathrm{C}^{34} \mathrm{~S}$, and $\mathrm{mm}$ continuum (from Kurtz et al. 2000), and $R_{X, Y}$ radius of the spherical region traced by $X, Y$. One can demonstrate that if Eq. (11) holds for the volume density at radius $R$, then it must hold also for the mean density inside $R$, and vice versa. This result is important because it makes possible to use the mean densities given in Table 9 to derive an estimate of $p$.

As already done for the temperature profile, we assume that $p$ is the same for all sources. Under this hypothesis, one can plot $\log \left(n_{X} / n_{Y}\right)$ versus $\log \left(\Theta_{X} / \Theta_{Y}\right)$ for all possible ratios and all sources: this is shown in Fig. 10. The correlation is excellent (the correlation coefficient is -0.97 ) and well reproduced by a linear fit with $p=-2.59 \pm 0.07$. This result is consistent with the density profiles predicted by star formation models (Shu et al. 1987; Li 1999) and supports the idea discussed in Sect. 4.1 that our clumps are marginally stable. Noticeably, in $\mathrm{W} 3\left(\mathrm{H}_{2} \mathrm{O}\right)-$ an object of the same type as those observed by us - an even steeper density law has been derived by Mauersberger et al. (1988): they find indirect evidence for $p$ ranging between -3 and -2.8 . This is in reasonable agreement with our finding.

It is worth noting that the previous density law applies to both the core (empty points in Fig. 10) and the surrounding clump (filled points) independently. This result 


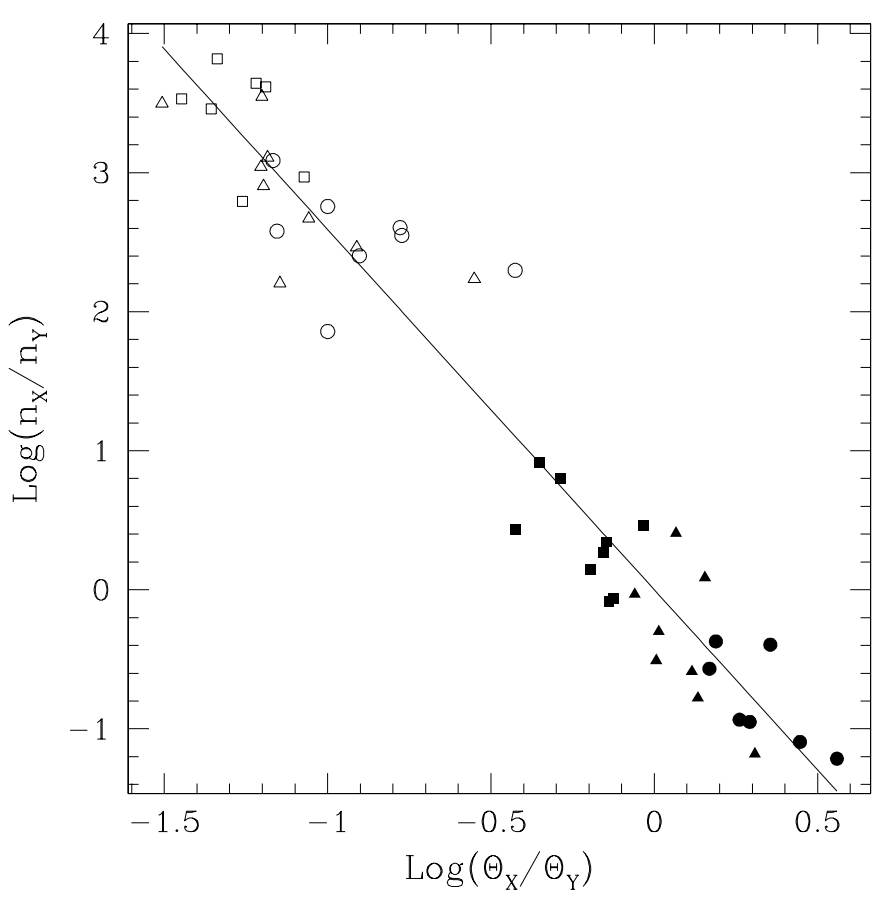

Fig. 10. Same as Fig. 9 for the $\mathrm{H}_{2}$ density. Filled symbols: circles correspond to ratios between densities from $\mathrm{C}^{17} \mathrm{O}$ and $\mathrm{C}^{34} \mathrm{~S}$, squares to ratios between densities from $\mathrm{C}^{34} \mathrm{~S}$ and $\mathrm{CH}_{3} \mathrm{C}_{2} \mathrm{H}$, and triangles to ratios between densities from $\mathrm{C}^{17} \mathrm{O}$ and $\mathrm{CH}_{3} \mathrm{C}_{2} \mathrm{H}$. Empty symbols: circles, triangles, and squares correspond respectively to ratios between densities estimated from Table 1 of Kurtz et al. (2000) and those derived from $\mathrm{C}^{17} \mathrm{O}, \mathrm{CH}_{3} \mathrm{C}_{2} \mathrm{H}$, and $\mathrm{C}^{34} \mathrm{~S}$. The straight line represents a least square fit of the type $n \propto \theta^{p}$, with $p=-2.59 \pm 0.07$. The correlation coefficient is -0.97 .

is not entirely consistent with the findings of Hatchell et al. (2000), who fit their continuum maps of similar clumps around UC HII regions with shallower density profiles. However, their fits are model dependent, whereas our results have been obtained under relatively simple assumptions, which in our opinion make them more reliable.

On the other hand, we know that massive stars have already formed inside the clumps, which means that gravitational collapse is going on in the central regions. According to Shu et al. (1987) this occurs within a radius $R_{2}(\mathrm{pc})=$ $1.72 \times 10^{-3} M_{*}\left(M_{\odot}\right)\left[\Delta v_{1 / 2}\left(\mathrm{~km} \mathrm{~s}^{-1}\right)\right]^{-2}$, where $M_{*}$ is the mass of the star created by the collapse: inside this radius the density should be $\propto R^{-3 / 2}$. In our case, the maximum value of $R_{2}$ is obtained for $M_{*} \simeq 60 M_{\odot}$ and $\Delta v_{1 / 2} \simeq 2 \mathrm{~km} \mathrm{~s}^{-1}$, which give $R_{2} \simeq 0.026 \mathrm{pc}$, much less than the minimum radius measured in any of the tracers used in Fig. 10: this confirms that the density profile measured with such tracers can be $\propto R^{-2}$, close to our result.

It is worth noting that $n \propto R^{-2}$ holds for an isothermal sphere, which seems to contradict the result $T \propto R^{-0.55}$ found in Sect. 4.2.1. However, the density gradient is established on dynamical timescales, much longer than the radiative timescale needed for the newly born high-mass stars to heat up the clumps. As a result, the density profile is a "remnant" of the primeval structure of the clump, whereas the temperature profile reflects the present heating by the embedded stars.

\subsubsection{Velocity structure}

Given the low angular resolution of our observations, the only possibility to investigate the velocity field in the clumps is to study the variation of the line shape as a function of the corresponding excitation energy: transitions from higher energy levels are expected to arise from inner regions since the temperature increases towards the center of the clumps, as previously discussed. This makes possible to sample the clumps at different radii by means of the different $K$ lines of $\mathrm{CH}_{3} \mathrm{C}_{2} \mathrm{H}$.

To this purpose, we have relaxed the assumption of equal line FWHM and peak velocity used in the line fits illustrated in Sect. 2.3 and we have fitted each $K$ component independently from the others. Then we have considered the variation of the line FWHM and peak velocity as a function of excitation energy. To this purpose, for each source we have computed the ratio between the FWHM of each line and that of a reference line, and the difference between the velocity of each line and that of the same reference line. The latter was the $(6-5) K=2$ transition, which is strong and does not overlap with other $K$ lines. Such ratios and differences have been plotted as a function of the ratio between the energies of the corresponding transitions and that of the reference line: the result is shown in Fig. 11. Clearly, no trend is seen for the velocity: this suggests that all $\mathrm{CH}_{3} \mathrm{C}_{2} \mathrm{H}$ lines trace the whole emitting region, as expected for optically thin transitions. On the contrary, the FWHM ratio shows a slight tendency to increase with the energy of the transition.

This fact cannot be explained with thermal broadening of the lines, because temperature changes at most from $\sim 10 \mathrm{~K}$ on the clump surface to $\sim 100 \mathrm{~K}$ in the inner regions: the corresponding contribution to the line width is respectively $0.1 \mathrm{~km} \mathrm{~s}^{-1}$ and $0.34 \mathrm{~km} \mathrm{~s}^{-1}$, which for a non-thermal line width of at least $\sim 3 \mathrm{~km} \mathrm{~s}^{-1}$ determines a variation of $0.6 \%$ of the line width. The latter is negligible to our purposes.

Another explanation for the observed line broadening at high energy might be an increase of optical depth for the high-energy transitions. It is possible to demonstrate that for an optical depth $\tau$ at the line centre, the ratio between the line FWHM, $\Delta v$, and the corresponding value in the optically thin limit, $\Delta v_{0}$ (i.e. the intrinsic line width), is given by the expression

$$
\frac{\Delta v}{\Delta v_{0}}=\sqrt{-\frac{1}{\ln 2} \ln \left[\frac{\ln 2-\ln \left(1+e^{-\tau}\right)}{\tau}\right]}
$$

From this, one can see that $\tau \simeq 4$ is needed to obtain $\Delta v / \Delta v_{0} \simeq 1.5$, i.e. the maximum ratio seen in the top panel of Fig. 11. As already discussed at length in Sects. 3.2 and 3.4.1, such a large value must refer to a very small region, which cannot contribute to the $\mathrm{CH}_{3} \mathrm{C}_{2} \mathrm{H}$ emission observed by us on a much larger scale. Therefore, 

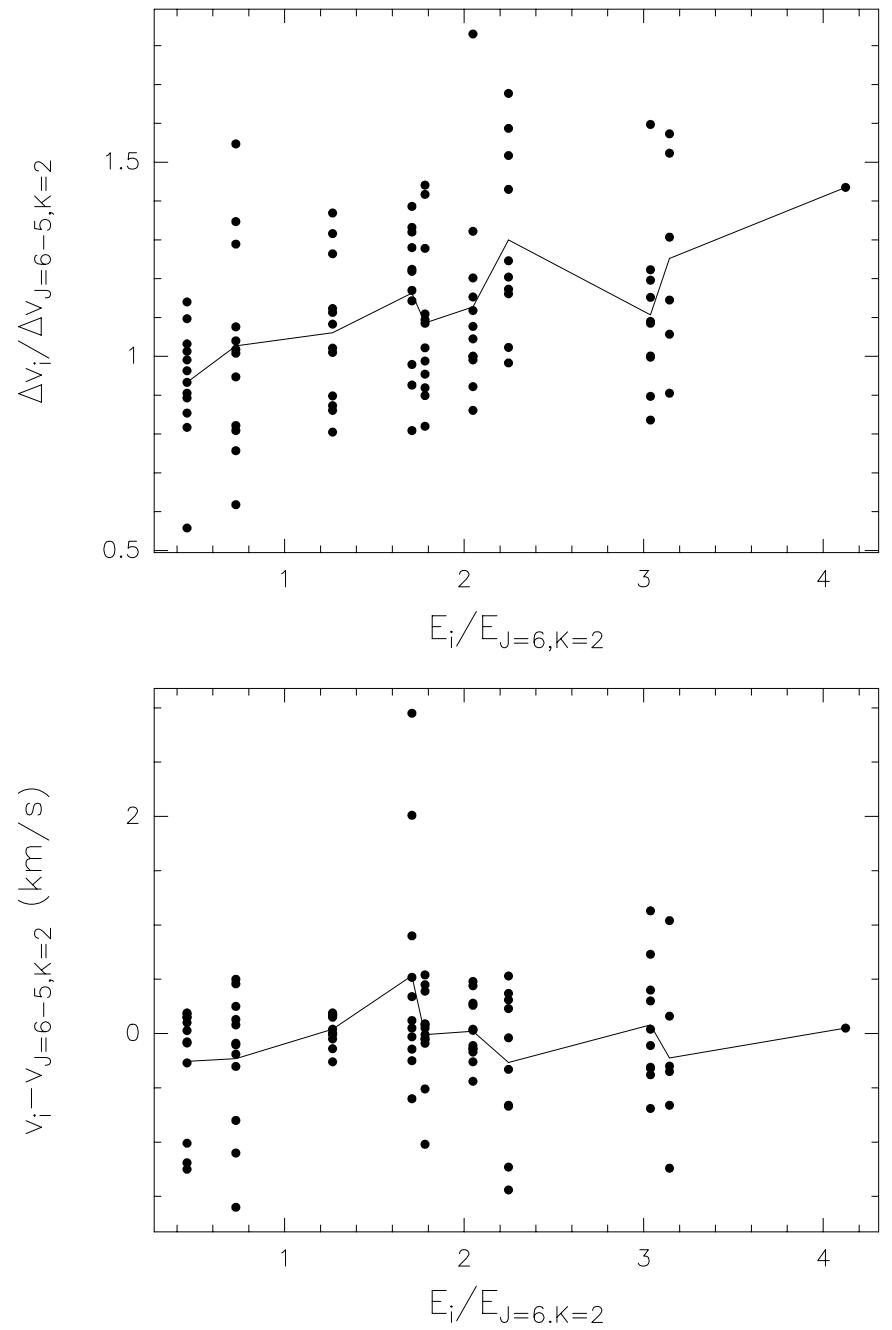

Fig. 11. Top panel: plot of the ratio between the FWHM of a $\mathrm{CH}_{3} \mathrm{C}_{2} \mathrm{H} K$ line and the FWHM of the $(6-5) K=2$ line, versus the corresponding excitation energy ratio. The line connects the mean values of the ratios obtained for each transition: one can see a slight increase of line width with energy. Bottom panel: same as top panel, for the difference in peak velocity between each $K$ line and the (6-5) $K=2$ line. Unlike the FHWM, no trend is present and the peak velocity is approximately the same for all transitions

line broadening is unlikely to be due to optical depth effects.

In conclusion, we believe that the most plausible explanation for Fig. 11 is that the velocity dispersion increases with energy of the transition and hence towards the centre of the clumps. Various mechanisms can explain this trend: keplerian rotation, infall motions, and turbulence caused by winds/outflows from the embedded, young high-mass stars. Although the present observations cannot discriminate among these, it is worth noting that the infall hypothesis is consistent with the scenario proposed in Sects. 4.1 and 4.2.2.

\section{Conclusions}

We have used the IRAM 30-m and FCRAO 14-m telescopes to observe a sample of $12 \mathrm{UC}$ HII regions in the $J=6-5,8-7$, and $13-12$ transitions of $\mathrm{CH}_{3} \mathrm{C}_{2} \mathrm{H}$. Emission has been detected in all of the objects and the following results have been obtained:

- we have estimated the diameters of the $\mathrm{CH}_{3} \mathrm{C}_{2} \mathrm{H}$ emitting regions by comparing the main beam brightness temperatures measured with the $30-\mathrm{m}$ and the FCRAO telescopes: a typical diameter turns out to be $\sim 0.9 \mathrm{pc}$, of the same order of those obtained with other medium density tracers (Cesaroni et al. 1991; Hofner et al. 2000).

- we have assessed that the $\mathrm{CH}_{3} \mathrm{C}_{2} \mathrm{H}$ molecule is in LTE and derived an accurate estimate of the temperature of the molecular clumps associated with these high-mass star forming regions: the temperatures range from 30 to $56 \mathrm{~K}$.

- we have derived $\mathrm{CH}_{3} \mathrm{C}_{2} \mathrm{H}$ column densities of $10^{14}$ $10^{15} \mathrm{~cm}^{-2}$ corresponding to $\mathrm{H}_{2}$ volume densities of $\sim 10^{5}-10^{6} \mathrm{~cm}^{-3}$.

- by comparing our results with those obtained by other authors using different tracers (Cesaroni et al. 1991; Hofner et al. 2000; Kurtz et al. 2000) we find that temperature and density depend on the distance from the clump centre, $R$, according to the relations $n \propto R^{-2.6}$ and $T \propto R^{-0.5}$ : these are consistent with marginally stable clumps heated by the embedded massive stars.

- the masses of the clumps turn out to be $\sim 3$ times greater than the corresponding virial estimates obtained ignoring magnetic fields and external pressure: we suggest that this might indicate that the clumps are on the verge of gravitational collapse and that magnetic fields might play an important role in stabilising the clumps; we find that the magnetic fields needed for virial equilibrium are of the order of $1 \mathrm{mG}$.

- the dynamical timescales on which the clumps may collapse are of $\sim 10^{5} \mathrm{yr}$ : these imply mass accretion rates of $\sim 10^{-2} M_{\odot} \mathrm{yr}^{-1}$.

- the line width shows a tendency to increase for increasing excitation energy, and hence for decreasing distance from the centre: a plausible explanation is that such a line broadening is due to collapse of the inner regions.

Acknowledgements. It is a pleasure to thank Malcolm Walmsley for critically reading the manuscript and Daniele Galli and Gianni Comoretto for stimulating discussions. The anonymous referee is also acknowledged for constructive criticisms that much improved the presentation.

\section{References}

Behrend, R., \& Maeder, A. 2001, A\&A, 373, 190

Bergin, A. E., Goldsmith, P. F., Snell, R. L., \& Ungerechts, H. 1994, ApJ, 431, 674 
Cesaroni, R., Hofner, P., Walmsley, C. M., \& Churchwell, E. 1998, A\&A, 331, 709

Cesaroni, R., Walmsley, C. M., Kömpe, C., \& Churchwell, E. 1991, A\&A, 252, 278

Cesaroni, R., Felli, M., Jenness, T., et al. 1999, A\&A, 345, 949

Chini, R., Kreysa, E., Mezger, P. G., \& Gemünd, H.-P. 1986, A\&A, 154, L8

Churchwell, E., Walmsley, C. M., \& Cesaroni, R. 1990, A\&AS, 83,119

Dubrulle, A., Boucher, D., Burie, J., \& Demaison, J. 1978, J. Mol. Spec., 72, 158

Erickson, N. R., Goldsmith, P. F., Novak, G., et al. 1992, IEEE Transactions on Microwave Theory and Techniques, 40, 1

Hatchell, J., Fuller, G. A., Millar, T. J., et al. 2000, A\&A, 357, 637

Hofner, P., Kurtz, S., Churchwell, E., Walmsley, C. M., \& Cesaroni, R. 1996, ApJ, 460, 359

Hofner, P., Wyrowski, F., Walmsley, C. M., \& Churchwell, E. 2000, ApJ, 536, 393

Hofner, P., Peterson, S., \& Cesaroni, R. 2000, ApJ, 514, 899

Hollis, J. M. 1982, ApJ, 260, 159

Hunter, T. R., Churchwell, E., Watson, C., et al. 2000, AJ, 119,271

Irvine, W. M., Goldsmith, P. F., \& Hjalmarson, H. 1987, Interstellar Processes, ed. D. J. Hollenbach, \& H. A. Thronson, 561

Kurtz, S., Cesaroni, R., Churchwell, E., Hofner, P., \& Walmsley, C. M. 2000, Protostars and Planets IV, ed. V. Mannings, A. Boss, \& S. Russel (Tucson: Univ. of Arizona Press)
Kutner, M. L., \& Ulich, B. L. 1981, ApJ, 250, 341

Lai, S., Crutcher, R. M., Girart, J. M., \& Rao, R. 2001, ApJ, 561,864

Li, Z. 1999, ApJ, 526, 806

MacLaren, I., Richardson, K. M., \& Wolfendale, A. W. 1988, ApJ, 333, 821

Mauersberger, R., Wilson, T. L., \& Henkel, C. 1988, A\&A, 201,123

Maxia, C., Testi, L., Cesaroni, R., \& Walmsley, C. M. 2001, A\&A, 371, 287

McKee, C. F., Zweibel, E. G., Goodman, A. A., \& Heiles, C. 1993, in Protostars and Planets III, ed. E. H. Levy, \& J. I. Lunine, 327

Mezger, P. G., Zylka, R., \& Wink, J. E. 1990, A\&A, 228, 35

Olmi, L., Cesaroni, R., \& Walmsley, C. M. 1993, A\&A, 276, 489

Olmi, L., \& Cesaroni, R. 1999, A\&A, 352, 266

Plume, R., Jaffe, D. T., Evans II, N. J., et al. 1997, ApJ, 476, 730

Shu, F. H., Adams, F. C., \& Lizano, S. 1987, ARA\&A, 25, 23

Spitzer, L. 1978, Physical processes in the interstellar medium (John Wiley \& sons, Inc.)

Townes, C. H., \& Schawlow, A. L. 1975, Microwave spectroscopy (Dover, New York)

Wilson, T. L., \& Rood, R. 1994, ARA\&A, 32, 191

Wood, D. O. S., \& Churchwell, E. 1989a, ApJS, 69, 831

Wood, D. O .S., \& Churchwell, E. 1989b, ApJ, 340, 265 